\title{
Zur Quantentheorie nichtlinearer Wellengleichungen III
}

\author{
Von W. Heisenberg, F. Kortel und H. Mitter \\ Aus dem Max-Planck-Institut für Physik, Göttingen \\ (Z. Naturforschg. 10a, 425-446 [1955]; eingegangen am 19. April 1955)
}

\begin{abstract}
Die vorliegende Arbeit gibt die mathematische Ausarbeitung eines Programms, das mit zwei früheren Arbeiten über ein Modell einer Theorie der Elementarteilchen aufgestellt worden war. Im ersten Teil der Arbeit wird die Verbindung zu den üblichen Methoden der Integralgleichungen hergestellt, im zweiten die Kräfte zwischen den Spinorteilchen berechnet und die Massen der Bose-Teilchen bestimmt. Im einzelnen umfaßt die Arbeit folgende Abschnitte: Einleitung: Die Axiomatik der neuen Quantisierungsmethode. 1. Beziehungen zwischen dem $\tau$-Gleichungssystem und der Methode der Integralgleichungen. a) Übergang von den $\tau$-Gleichungen $\mathrm{zu}$ Integralgleichungen und Darstellung durch Graphen. b) Streuprobleme. c) Spinorteilchen. 2. Wechselwirkung und gebundene Zustände. a) Kraft zwischen zwei Spinorteilchen. b) Bose-Teilchen der Ruhmasse Null. c) Höhere Näherungen für die Kraft zwischen zwei Spinorteilchen und Berechnung der ,Feinstrukturkonstante“. d) Bose-Teilchen nichtverschwindender Ruhmasse. e) Die Integralgleichungen für die Funktion $S_{\mathrm{F}}$. Das Eigenwertspektrum, das sich aus der einfachen Ausgangsgleichung ergibt, hat eine überraschende Ähnlichkeit mit dem Massenspektrum der wirklichen Elementarteilchen. Insbesondere sind auch wesentliche Teile der Elektrodynamik in der Ausgangsgleichung enthalten.
\end{abstract}

$\mathrm{I}$ n zwei früheren Arbeiten ${ }^{1}$ wurde als ein Modell für eine zukünftige Theorie der Elementarteilchen die Wellengleichung

$$
\gamma_{\nu} \frac{\partial \psi}{\partial x_{\nu}}+l^{2} \psi\left(\psi^{+} \psi\right)=0
$$

studiert und der Versuch unternommen, sie nach einer neuartigen Methode zu quantisieren.

Der wesentliche Zug dieser Methode bestand in der Annahme, daß, ähnlich wie in der linearen Theorie oder der Quantenmechanik, ein enger $\mathrm{Zu}$ sammenhang existieren soll zwischen der quantentheoretischen Vertauschungsrelation und der Fortpflanzungsfunktion, die sich als Lösung der klassischen Wellengleichung (1) ergibt. Diese Annahme, über deren Durchführbarkeit erst die weiteren Rechnungen entscheiden können, hat zur Folge, daß die Vertauschungsrelation - im Gegensatz zur linearen Theorie - auf dem Lichtkegel keine $\delta$ Funktion enthalten kann, sondern dort unendlich oszilliert. Dadurch werden grundsätzliche Änderungen im Aufbau der Theorie notwendig; denn es ist seit längerer Zeit bekannt ${ }^{2}$, daß in $\operatorname{dem} \mathrm{Va}$ kuumerwartungswert der Vertauschungsrelation

$$
\begin{aligned}
\sum_{\Phi}\left\{\langle\Omega|\psi(x)| \Phi\rangle\left\langle\Phi\left|\psi^{+}\left(x^{\prime}\right)\right| \Omega\right\rangle\right. \\
\left.+\left\langle\Omega\left|\psi^{+}\left(x^{\prime}\right)\right| \Phi\right\rangle\langle\Phi|\psi(x)| \Omega\rangle\right\}
\end{aligned}
$$

${ }^{1}$ W. Heisen berg, Nachr. d. Göttinger Akad. Wiss. 1953, S. 111 und Z. Naturforschg. 9a, 292 [1954], im folgenden als l. c. I und II zitiert. jeweils die Teilgesamtheit von stationären $\mathrm{Zu}$ ständen $\Phi$, die zu einem diskreten Masseneigenwert gehört, eine $\delta$-Funktion auf dem Lichtkegel beiträgt und daß alle $\delta$-Funktionen der verschiedenen Masseneigenwerte sich mit dem gleichen Vorzeichen addieren.

Um der neuen Vertauschungsfunktion einen Sinn zu geben, wurde daher an Stelle des HilbertRaums der gewöhnlichen Theorie ein in zwei Teile zerfallender Hilbert-Raum mit folgenden Eigenschaften postuliert:

Der Hilbert-Raum I enthält alle Zustände bis $\mathrm{zu}$ einer sehr großen Grenzmasse $M_{\mathrm{g}}$, so daß alle physikalisch überhaupt realisierbaren Zustände zum Hilbert-Raum I gehören. Im Hilbert-Raum I gelten alle Regeln der üblichen Quantentheorie. Daneben gibt es einen Hilbert-Raum II, der nur virtuellen oder symbolischen Charakter hat und Zustände anderer Art umfaßt. In diesem HilbertRaum sind weder die Massen, Energien und Impulse der Zustände definiert (abgesehen von der Fiktion, daß alle Massen oberhalb von $M_{\mathrm{g}}$ liegen sollen) noch die Übergangselemente der Operatoren $\psi(x)$ oder $\psi^{+}(x)$ zu einem derartigen $\mathrm{Zu}$ stand; die Regeln der Quantentheorie können in diesem Raum nicht angewendet werden. Wohl aber

${ }^{2}$ H. Lehmann, Nuovo Cim. 11, 342 [1954]; G. Källén, Helv. Phys. Acta 25, 417 [1952]. 
wird der pauschale Beitrag der Zustände des Hilbert-Raumes II, die als virtuelle $Z$ wischenzustände auftreten, zu Produkten von $\psi(x)$-Operatoren definiert. Es genügt dabei, Produkte von nur zwei solchen Operatoren zu betrachten, da Produkte von mehreren Operatoren sich auf solche von zwei zurückführen lassen und kompliziertere Funktionen von $\psi(x)$-Operatoren, sofern sie überhaupt vorkommen, nur als Potenzreihen definiert sein sollen.

Bei Produkten von zwei $\psi(x)$ - bzw. $\psi^{+}(x)$-Operatoren sollen die als $Z$ wischenzustände $\Phi_{\mathrm{z}}$ auftretenden Zustände des Hilbert-Raumes II überhaupt keinen Beitrag liefern in folgenden Fällen:

a) Wenn Anfangs- und Endzustand im HilbertRaum I orthogonal zueinander sind; also z. B. in dem Produkt

$$
\begin{array}{r}
\sum_{\Phi_{\llcorner}}\left\langle\Phi_{\mathrm{a}}\left|\psi^{\prime}(x)\right| \Phi_{\mathrm{z}}\right\rangle\left\langle\Phi_{\mathrm{z}}\left|\psi^{+}\left(x^{\prime}\right)\right| \Phi_{\mathrm{e}}\right\rangle \text { dann, } \\
\text { wenn }\left\langle\Phi_{\mathrm{a}}, \Phi_{\mathrm{e}}\right\rangle=0,
\end{array}
$$

oder wenn $\Phi_{\mathrm{a}}$ oder $\Phi_{\mathrm{e}}$ selbst im Hilbert-Raum II liegen.

b) In der Vertauschungsrelation

$$
\begin{aligned}
\sum_{\psi_{z}}\left\{\left\langle\Phi|\psi(x)| \Phi_{\mathrm{z}}\right\rangle\left\langle\Phi_{\mathrm{z}}\left|\psi^{+}\left(x^{\prime}\right)\right| \Phi\right\rangle\right. & \left.+\left\langle\Phi\left|\psi^{+}\left(x_{6}^{\prime}\right)\right| \Phi_{\mathrm{z}}\right\rangle\left\langle\Phi_{\mathrm{z}}|\psi(x)| \Phi\right\rangle\right\}
\end{aligned}
$$

dann, wenn $s=\Sigma\left(x-x^{\prime}\right)_{\nu}{ }^{2} \neq 0$.

Wenn jedoch in der Vertauschungsrelation $s=0$ ist, dann sollen die Zwischenzustände des HilbertRaums II einen Beitrag liefern, und zwar sollen sie gerade die $\delta$-Funktionen der Zwischenzustände aus dem Hilbert-Raum I kompensieren und statt dessen das unendlich oszillierende Verhalten der Fortpflanzungsfunktionen hervorbringen.

Wenn Anfangs- und Endzustand gleich sind, es sich aber nicht um die Vertauschungsrelation handelt, so liefern die Zwischenzustände des HilbertRaums II sogar dann einen Beitrag, wenn $s \neq 0$ ist. Betrachtet man insbesondere die Differenz

$$
\begin{aligned}
\sum_{\Phi_{z}}\left\{\left\langle\Phi|\psi(x)| \Phi_{\mathrm{z}}\right\rangle\left\langle\Phi_{\mathrm{z}}\left|\psi^{+}\left(x^{\prime}\right)\right| \Phi\right\rangle\right. & \left.-\left\langle\Phi\left|\psi^{+}\left(x^{\prime}\right)\right| \Phi_{\mathrm{z}}\right\rangle\left\langle\Phi_{\mathrm{z}}|\psi(x)| \Phi\right\rangle\right\},
\end{aligned}
$$

so sollen in diesem Ausdruck die Beiträge der Zwischenzustände II zu den Beiträgen in (4) in der gleichen Relation stehen wie in der gewöhnlichen Theorie die $S_{1}$-Funktion zur $S$-Funktion. Denn die

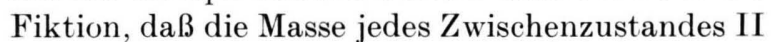
höher sein soll als die des Anfangs- und Endzustandes, erzwingt auf Grund der Regeln der Matrixmultiplikation, daß der Beitrag zu (5) durch den zu (4) festgelegt ist.
Insbesondere gilt also für den Vakuumerwartungswert der Vertauschungsrelation die Schwingersche Beziehung:

$$
S_{1}(r, t)=\frac{1}{\pi} \int_{-\infty}^{+\infty} \frac{\mathrm{d} t^{\prime}}{t^{\prime}} S\left(r, t-t^{\prime}\right) .
$$

Approximiert man die Vertauschungsrelation für $s \neq 0$ durch den Beitrag der zum tiefsten Masseneigenwert $x$ eines Fermions gehörigen Zustände, so ergibt sich damit

$$
\begin{aligned}
& S_{1}\left(x-x^{\prime}\right) \approx \frac{x^{2}}{4 \pi} \gamma_{\mu} \frac{\partial}{\partial x_{\mu}} \\
& \text {. } \mathrm{m}\left\{\frac{H_{1}(1)(\varkappa \sqrt{s})}{\varkappa \sqrt{s}}+\frac{2 i}{\pi \varkappa^{2} s}-\frac{i}{\pi} \lg \frac{\gamma \varkappa \sqrt{s}}{2}\right\} \\
& -\frac{\varkappa^{3}}{4 \pi} \Im \mathfrak{m}\left\{\frac{H_{1}{ }^{(1)}(\varkappa \sqrt{s})}{\varkappa \sqrt{s}}+\frac{2 i}{\pi \varkappa^{2} s}\right\} \text { für } s \neq 0 .
\end{aligned}
$$

$S_{1}(0)$ ist zunächst unbestimmt, da die Funktion $S(s)$ ursprünglich bei $s=0$ unendlich oszilliert, soll aber in allen Rechnungen durch den ,Mittelwert"

$$
S_{1}(0)=S(0)=0
$$

ersetzt werden. Damit ist die Rolle des HilbertRaums II vollständig festgelegt.

Zur Erläuterung dieser Rolle kann man noch folgende Überlegung anführen: Der Hilbert-Raum I müßte für die Definition der Theorie dann genügen, wenn alle in derTheorie vorkommenden Funktionen durch Entwicklung nach einem Orthogonalsystem (z.B. nach Fourier-Reihen) approximiert werden könnten. Das ist aber sicher nicht der Fall. Denn zwar wird man dies für alle physikalisch realisierbaren Wellenfelder annehmen dürfen; aber die in der mathematischen Darstellung vorkommende Vertauschungsfunktion und die mit ihr eng zusammenhängende Fortpflanzungsfunktion, deren fundamentale Rolle in der Theorie einleuchtet, lassen sich in der Nähe des Lichtkegels wegen der unendlichen Oszillationen nicht mehr durch Entwicklung nach einem Orthogonalsystem approximieren - während in der linearen Theorie die Fourier-Entwicklung der $\delta$-Funktion noch in einem gewissen Sinne möglich ist. Es ist also nicht weiter merkwürdig, daß das besondere Verhalten der Fortpflanzungsfunktion in der Nähe des Lichtkegels eine Erweiterung des Hilbert-Raums notwendig macht. Erst nach der ,,Mittelungsannahme" $S_{1}(0)=S(0)=0$ kann auch die Vertauschungsfunktion durch ein Fourier-Integral dargestellt werden.

Summiert man in der Vertauschungsrelation nur über die Zwischenzustände des Hilbert-Raumes I, 
so entsteht wie in der üblichen Theorie eine $\delta$ Funktion auf dem Lichtkegel und für $t=t^{\prime}=0$ ergibt sich bis auf einen konstanten Faktor die räumliche $\delta$-Funktion. In der gewöhnlichen Theorie wird dieser Faktor gleich der Einheit gesetzt, was zur sogenannten Thomas-Kuhnschen Summenregel führt. Auch hier kann der Faktor in dieser Weise festgesetzt werden, und tatsächlich sollen die späteren Rechnungen unter dieser Voraussetzung durchgeführt werden. Es muß aber hervorgehoben werden, daß die Fixierung des Faktors keine wesentliche physikalische Einschränkung bedeutet. Denn jede Änderung des Faktors könnte durch eine entsprechende Änderung der Kopplungskonstante $l$ in Gl. (1) kompensiert werden, so daß das Eigenwertspektrum das gleiche bleiben kann. Eine Änderung des Faktors vor der $\delta$-Funktion verursacht also nur eine physikalisch uninteressante Maßstabsveränderung.

Erst die Hinzunahme des Hilbert-Raums II ermöglicht die Beseitigung der $\delta$-Funktion auf dem Lichtkegel. Das Fehlen der $\delta$-Funktion scheint ganz allgemein eine notwendige Voraussetzung für die Konvergenz einer nichtlinearen Feld theorie zu sein; diese Vermutung wird insbesondere durch die neueren Untersuchungen von Källén und $\mathrm{Pauli}^{3}$ nahegelegi. In den üblichen Theorien kann die Beseitigung der $\delta$-Funktion nur durch Abweichungen von der relativistischen Kausalität (z. B. nicht hermitesche Hamilton-Funktion) erkauft werden. Die geschilderten Annahmen über den Hilbert-Raum II stellen den Versuch dar, diese Abweichungen ganz auf die Funktion $S_{1}$ zu schieben, wo sie erst gleichzeitig mit der Quantisierung, d. h. zusammen mit dem Teilchenbegriff, auftreten und als endliche Größe oder Kraft endlicher Reichweite der Elementarteilchen interpretiert werden können. Tatsächlich fällt die Funktion $S_{1}$ in Gl. (7) sehr viel langsamer ab als die $S_{1}$-Funktionen der üblichen Theorie. Während die $S_{1}$-Funktionen der üblichen Theorie für große $s$ bei endlicher Masse exponentiell, bei der Ruhmasse Null wie $s^{-3 / 2}$ abfallen, findet hier die Abnahme wie $s^{-1 / 2}$ statt. Dieser langsame Abfall stellt sozusagen den Mindestpreis dar, der für die Beseitigung der $\delta$-Funktion zu entrichten ist, und es ist daher wichtig, seine Konsequenzen im Rahmen der Theorie zu verfolgen. Die folgenden Untersuchungen greifen noch nicht das mathematisch sehr schwierige Problem an, die

${ }^{3}$ G. Källén u. W. Pauli, im Erscheinen. widerspruchsfreie Durchführbarkeit der geschilderten Ansätze streng zu beweisen, aber sie streben eine Aufklärung der von der üblichen Theorie abweichenden Züge des vorliegenden Versuchs an, die mit dem langsamen Abfall der $S_{1}$-Funktion zusammenhängen.

In der normalen Theorie besteht eine enge Verbindung zwischen den $S_{1}$-Funktionen und den zwischen zwei Elementarteilchen wirkenden Kräften. Wenn z. B. die Kraft zwischen zwei Teilchen durch ein bestimmtes Feld $\chi(x)$ übertragen wird, so gibt die zu diesem Feld $\chi(x)$ gehörige $S_{1}$ - bzw. $\Delta_{1}$-Funktion an, wie die Kraft vom Abstand zwischen den beiden Teilchen abhängt. Am deutlichsten kommt dieser Zusammenhang in dem Feynmanschen Graphenschema zum Ausdruck; die Wechselwirkung wird in diesem Schema durch eine oder mehrere $\chi$-Feldlinien dargestellt, die von der Linie des einen Teilchens $\mathrm{zu}$ der des anderen laufen. Für diese Linien ist jeweils die $S_{\mathrm{F}^{-}}$bzw. $\Delta_{\mathrm{F}^{-}}$-Funktion des $\chi$-Feldes einzusetzen, deren raumartiger Anteil eben die $S_{1}$ - bzw. $\Delta_{1}$-Funktion ist.

Der langsame Abfall der $S_{1}$-Funktion in der hier untersuchten Theorie deutet also darauf hin, daß es in ihr Kräfte großer Reichweite gibt - im Ge. gensatz zu einer Vermutung, die in l. c. II, S. 302 ausgesprochen wurde. Rechnet man die Kräfte zwischen zwei Elementarteilchen unter Benutzung der Gl. (7) nach dem Graphenschema aus (was in Kap. 2 geschehen soll), so ergeben sich in erster Näherung tatsächlich Kräfte vom Coulombschen Typus, d.h. die Wechselwirkungsenergie nimmt bei großen Abständen umgekehrt proportional dem Abstande ab.

Allerdings ist die Grundlage einer solchen Berechnung hier zunächst zweifelhaft, da die übliche Theorie von der Entwicklung nach einem kleinen Kopplungsparameter ausgeht, während hier von einer kleinen Kopplung gar nicht die Rede sein kann. Das durch den langsamen Abfall der $S_{1^{-}}$ Funktion gestellte Problem kann also erst angegriffen werden, wenn es gelingt, das in l. c. I und II zur Definition der Quantisierung benützte ,,neue Tamm-Dancoff-Verfahren" so auszubauen oder umzuformen, daß es mit den üblichen Integralgleichungsmethoden in Verbindung gebracht werden kann oder sich unmittelbar auf das Problem der Kräfte anwenden läßt. Das erste Kapitel der vorliegenden Arbeit soll also den Zusammenhang zwischen der in I und II benützten neuen TammDancoff-Methode und dem Integralgleichungs- 
system behandeln. Die Frage nach der Güte einer bestimmten Approximation ist allerdings auch damit noch nicht befriedigend beantwortet, weil die verwendeten Reihen, gleichviel in welcher Methode, bestenfalls wie numerische Reihen etwa vom Typus $1+\frac{1}{2}+\frac{1}{6}+\ldots$ konvergieren; denn es gibt grundsätzlich keinen kleinen Kopplungsparameter, nach dem entwickelt werden könnte. Aber das in I behandelte Problem des anharmonischen Oszillators mit starker Kopplung kann als Hinweis darauf betrachtet werden, daß die nach wenigen Gliedern abgebrochenen numerischen Reihen unter Umständen doch schon eine recht gute Näherung ergeben. Um wenigstens ein gewisses Kriterium für die Genauigkeit der gewonnenen Resultate zu erhalten, soll die Masse der leichtesten Fermi-Teilchen und damit die Konstante $x$ in Gl. (7) nach verschiedenen unabhängigen Methoden berechnet werden.

Erst im zweiten Kapitel werden dann die Kräfte zwischen zwei Elementarteilchen nach dem im ersten Kapitel entwickelten Verfahren behandelt. Die Existenz der Kräfte langer Reichweite vom Coulomb-Typus ergibt sich dabei zwangsläufig aus dem System der Integralgleichungen. Da sich jedes Kraftfeld, dessen erzeugendes Teilchen starke Beschleunigungen erfährt, als Strahlung von diesem Teilchen lösen und in den Raum wandern kann, schließt man aus den Kräften vom CoulombTypus auf die Existenz von Bose-Teilchen der Ruhmasse Null. Daß solche Teilchen als Eigenwerte der Tamm-Dancoff-Gleichungen tatsächlich auftreten, soll in Kapitel 2 ebenfalls nachgerechnet werden. Es wird sich dabei herausstellen, daß es nur vektorielle Teilchen der Ruhmasse Null gibt. Diese entsprechen in ihren Transformationseigenschaften genau den Lichtquanten, deren mathematische Formulierung hier also mit gewissen Einschränkungen als eine Neutrinotheorie des Lichtes bezeichnet werden kann. Am Schluß des zweiten Kapitels werden die Massen der leichtesten BoseTeilchen, deren Ruhmasse von Null verschieden ist, in der niedrigsten Näherung berechnet.

\section{Beziehungen zwischen dem $\tau$-Gleichungssystem und der Methode der Integralgleichungen}

a) Übergang von den $\tau$-Gleichungen $\mathrm{zu}$ Integralgleichungen und Darstellung durch Graphen

Die Operatorgleichung (1) stellt nach den Überlegungen von I und II ganz allgemein eine Diffe- rentialbeziehung zwischen einer $\tau$-Funktion und einer anderen $\tau$-Funktion mit einer um zwei größeren Variabelnzahl her. Integriert man diese Differentialgleichung mit Hilfe der Greenschen Funktion $G_{\alpha \beta}\left(x-x^{\prime}\right)$ der masselosen Dirac-Gleichung (hier und im folgenden ist bei den Funktionen $G_{\alpha \beta}\left(x-x^{\prime}\right)$ und $S_{\alpha \beta}\left(x-x^{\prime}\right)$ stets die Funktion $G_{\mathrm{F}}$ bzw. $S_{\mathrm{F}}$ gemeint, sofern der Index nicht besonders angeschrieben wird; dann ist die Feynmansche Frequenzbedingung erfüllt), so erhält man eine Darstellung der $\tau$-Funktion der niederen Variabelnzahl durch die Faltung der Greenschen Funktion mit der $\tau$-Funktion der um zwei höheren Variabelnzahl. Durch mehrmalige Anwendung dieses Rechenprozesses kann man die $\tau$-Funktion auch durch $\tau$-Funktionen ausdrücken, deren Variabelnzahl um vier, sechs oder schließlich irgendeine gerade Zahl höher ist als die der Ausgangsfunktion. Nach den in I und II besprochenen Vorschriften bricht man dieses Gleichungssystem dann bei einer bestimmten Variabelnzahl $N$ ab, indem man die $\tau$-Funktion mit $N+2$ Variabeln durch die $\varphi$-Funktionen der Variabelnzahl $N+2, N, N-2, N-4, \ldots$. ausdrückt und die $\varphi$-Funktion der höchsten Variabelnzahl $\mathrm{N}+2$ gleich Null setzt. Durch diese Festsetzung ist das Gleichungssystem der TammDancoff-Methode vollständig festgelegt.

In dieser Form kann das Gleichungssystem aber nur in den einfachsten Fällen behandelt werden; meistens wird man das System durch weitere Vernachlässigungen vereinfachen müssen, um es einer Behandlung zugänglich zu machen. Die nächstliegende Vereinfachung besteht in der Annahme, daß man bei der Darstellung von $\tau(N+2)$ nicht nur $\varphi(N+2)=0$ setzt, sondern auch noch die Glie$\operatorname{der} \varphi(N), \varphi(N-2), \varphi(N-4), \ldots$ vernachlässigt ${ }^{4}$, bis man zu der Variabelnzahl der Ausgangsfunktion kommt, die mindestens gleich der Zahl der an dem Zustand beteiligten Teilchen sein muß. Die Ausgangsfunktion und noch niedrigere $\varphi$-Funktionen kann man dann wieder durch $\tau$-Funktionen ausdrücken und erhält in dieser Weise Integralgleichungen, in denen nur die $\tau$-Funktionen niedriger Variabelnzahl vorkommen. Das so entstehende Gleichungssystem ist allerdings erheblich ungenauer als das System der $\tau$-Gleichungen mit der Annahme $\varphi(N+2)=0$, mag aber für eine qualita-

${ }^{4}$ In l. c. II war an dieser Stelle irrtümlich von einer. Vernachlässigung der Funktionen $\tau(N-2)$ usw. gesprochen worden. 
tive Übersicht über das Verhalten der Lösungen noch brauchbar sein. Die in dieser Weise gebildeten Integralgleichungen sind den Integralgleichungen der üblichen Theorie, z.B. der Bethe-SalpeterGleichung eng verwandt, sind aber nicht genau identisch mit ihnen. Unterschiede ergeben sich hauptsächlich an folgenden Stellen: In der üblichen Theorie ist die Kontraktionsfunktion $S_{\mathrm{F}}$ mit der Greenschen Funktion $G_{\mathrm{F}}$ identisch. Das ist hier nicht der Fall. In den $\tau$-Funktionsgleichungen der gewöhnlichen Theorie treten auf der rechten Seite $\delta$-Funktionen auf, die von der Umordnung der Faktoren im $T$-Produkt bei Änderung der Zeitreihenfolge herrühren. Diese $\delta$-Funktionen fallen hier weg. Die Vernachlässigung der $\varphi$-Funktionen mit höherer Variabelnzahl als der der Ausgangsfunktionen läßt sich in der gewöhnlichen Theorie damit begründen, daß ihre Berücksichtigung bei Lösung dèr Gleichung mit Hilfe der Neumannschen Reihe zwar möglich wäre, aber nur zu Gliedern mit einer höheren Potenz des Kopplungsparameters führen würde, von denen in der behandelten Näherung doch nicht alle ausgerechnet werden können. Diese Begründung ist hier nicht mehr möglich, da es sich nicht mehr um die Entwicklung nach einem kleinen Kopplungsparameter handelt. Man kann nur versuchsweise annehmen, daß die $\varphi$-Funktionen höherer Variabelnzahl kleiner werden als die niedriger Variabelnzahl im Sinne einer konvergenten Zahlenreihe; tatsächlich beträgt z. B. bei dem in I durchgerechneten Beispiel des anharmonischen Oszillators mit starker Kopplung $\varphi(3)$ nur etwa ein Fünftel von $\varphi(1)$. Erst nachträglich läßt sich also die Berechtigung der Annahme am Ergebnis kontrollieren.

Die beschriebenen Integralgleichungen lassen sich durch ,Graphen“ darstellen. Wir nehmen zu diesem Zweck an, daß für die $\tau$-Funktionen der niedrigsten Ordnung kein Unterschied zwischen den $\tau$-Funktionen und den $\varphi$-Funktionen besteht. Diese Voraussetzung genügt für die Berechnung der Masse der einfachsten Fermi- und Bose-Teilchen sowie für die Behandlung der Kräfte zwischen zwei Teilchen. Die Variabeln in der Ausgangs-

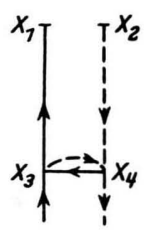

Abb. 1. funktion [als Beispiel: $\tau\left(x_{1} \mid x_{2}\right)$ ] bezeichnen wir als Endpunkte und zeichnen sie ans obere Ende des Graphen (Abb.1). Geht man nun durch Anwendung der Greenschen Funktion auf eineVariable, z. B. $x_{1}$, zueiner $\tau$-Funktion mitzweiweiterenVariabeln über, so steht in dieser neuen $\tau$-Funktion statt $x_{1}$ dreimal eine neue Variable $x_{3}$ [im Beispiel: $\tau\left(x_{3} x_{3} \mid x_{3} x_{2}\right)$ ]. Wir zeichnen also zur Darstellung dieses Schrittes eine ,G-Linie“ (in der Figur ausgezogen) von $x_{1}$ nach $x_{3}$. Durch Anwendung der Greenschen Funktion auf irgendeine der neuen Variabeln entsteht eine $\tau$-Funktion mit zwei weiteren Variabeln [im Beispiel: $\left.\tau\left(x_{3} x_{4} x_{4} \mid x_{4} x_{3} x_{2}\right)\right]$; dieser Schritt wird durch eine $G$-Linie von $x_{3}$ nach $x_{4}$ dargestellt usw. Schließlich wird die höchste $\tau$-Funktion [im Beispiel: $\left.\tau\left(x_{3} x_{4} x_{4} \mid x_{4} x_{3} x_{2}\right)\right]$ durch $\varphi$-Funktionen dargestellt, wobei alle $\varphi$-Funktionen mit mehr Variabeln als die Ausgangsfunktion (im Beispiel: mit mehr als zwei Variabeln) weggelassen werden. Die Kontraktionsfunktionen werden als ,S-Linien“ (in der Abb. gestrichelt gezeichnet) in das Diagramm eingetragen. Die Variabeln der $\phi$-Funktion (im Beispiel: $x_{3}$ und $x_{4}$ ), die hier mit der $\tau$-Funktion identisch ist, werden als Anfangspunkte bezeichnet. In der Figur kann auch noch in der üblichen Weise durch eine Pfeilrichtung angedeutet werden, ob die Operation an einer Funktion $\psi(x)$ oder $\psi^{+}(x)$ ausgeführt wird. In dieser Weise wird durch den Graphen eine Integralgleichung für die Ausgangsfunktion festgelegt (man kann sich den Graphen periodisch als ,Leiter" fortgesetzt oder als im Ring geschlossen denken).

Aus der eben gegebenen Herleitung folgen für die zulässigen Graphen die folgenden Regeln :

1. In jedem Punkt des Graphen, ausgenommen die Endpunkte, treffen sich vier Linien, von deren Pfeilrichtungen zwei vom Punkt weg, zwei auf ihn zu weisen.

2. In dem zwischen Anfangs- und Endpunkten aufgespannten Liniensystem gibt es gleich viele $G$ - und $S$-Linien.

3. Jeder Punkt ist durch einen (und nur einen) $G$-Linienzug mit einem der Endpunkte verbunden.

4. Die Zahl der Punkte (Endpunkte werden nicht mitgezählt) im Graphen ist $(N+1) / 2$ für ungerade $N$ und $N / 2$ für gerade $N$.

5. Bei der Darstellung des Kerns der Integralgleichung entspricht jeder G-Linie der Ausdruck $\frac{-i}{2} l^{2} G_{\mathrm{F}}$, jeder $S$-Linie der Ausdruck $\frac{1}{2} S_{\mathrm{F}}$. Die Verbindung zweier Linien gleicher Richtung an einem Punkt zu einem Linienzug bedeutet Matrixmultiplikation der zugehörigen Funktionen.

6. Der Kern der Integralgleichung ist festgelegt, wenn die Punkte des Graphen, die Richtungen an den Endpunkten und die $G$-Linien mit ihren Rich- 
tungs- und Zusammenhangsverhältnissen gegeben sind (Beispiel: Abb. 2). Die S-Linien können dann im allgemeinen auf mehrere Weisen so

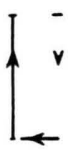
eingetragen werden, daß von Anfangs- zu Endpunkten durch gehende oder geschlossene Linienzüge entstehen. Über diese verschiedenen Möglichkeiten ist im Kern-

Abb. 2. der Integralgleichung zu summieren (Beispiel: Abb. 3).

Dabei kann das Vorzeichen der verschiedenen Beiträge bestimmt werden, indem man zunächst bis zur höchsten $\tau$-Funktion die Reihenfolge der

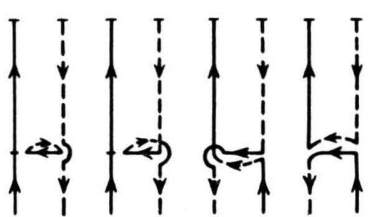

Abb. 3. Variabeln ungeändert läßt und dann in der höchsten $\tau$-Funktion die zuS-Funktionen zu kontrahierendenPaare markiert und abzählt, wie viele Vorzeichenwechsel bei der Kontraktion auftreten.

In jeder Näherung kann der $G$-Linienzug noch auf mehrere Weisen festgelegt werden. In dem angeführten Beispiel sind neben Abb. 2 noch die sechs in Abb. 4 angegebenen G-Linienzüge zulässig.

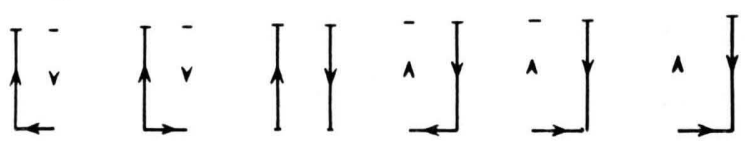

Abb. 4.

Man erkennt daraus, daß man für die gleiche $\tau$ Funktion eine Reihe verschiedener Integralgleichungen erhält, die wahrscheinlich nicht genau das gleiche aussagen, aber doch etwa die gleiche Genauigkeit beanspruchen können. Man kann durch Mittelbildung zwischen verschiedenen Kernen dieser Integralgleichungen zu neuen derartigen Gleichungen kommen, die auch wieder näherungsweise gelten sollten.

Es liegt daher nahe, eine solche Mittelbildung durchzuführen, daß die entstehende Gleichung den aus der Bethe-Salpeter-Gleichung gewonnenen Systemen möglichst ähnlich wird, da man vielleicht erwarten darf, daß man in der betreffenden Näherung damit die relativ beste Approximation erhält. In einfachen Fällen bedeutet dies, daß man über die verschiedenen $G$-Linienzüge mit gleichen Gewichten mitteln muß; in komplizierteren Problemen müssen die Möglichkeiten der Angleichung an die Bethe-Salpeter-Cleichung ausführlicher untersucht werden.

\section{b) Streuprobleme}

Nach dem bisher geschilderten Verfahren läßt sich auch die Streuung von Teilchen an anderen Teilchen behandeln. Die Beziehungen, die sich dabei zu den Verfahren der üblichen Theorie ergeben, sollen an dem Peispiel der gegenseitigen Streuung von zwei Teilchen näher ausgeführt werden.

Wir betrachten also die Funktion

$$
\tau\left(x_{1} x_{2}\right)=\varphi\left(x_{1} x_{2}\right)
$$

und suchen die zugehörige Integralgleichung auf. Nachdem die Näherung durch eine Annahme über $N$ festgelegt ist, wählen wir unter den nach 1 a) möglichen Graphen solche aus, bei denen ein $G$ Linienzug vom Anfangspunkt $x_{1}{ }^{\prime}$ zum Endpunkt $x_{1}$ und ein zweiter $G$-Linienzug von $x_{2}^{\prime}$ zum Endpunkt $x_{2}$ führt. Fin Beispiel dafür ist in Abb. 5 angegeben (für $N=12$ ). Den Kern $K$ der Integralgleichung

$$
\begin{aligned}
\varphi\left(x_{1} x_{2}\right) & =K \varphi\left(x_{1}{ }^{\prime} x_{2}{ }^{\prime}\right) \\
& =\int K\left(x_{1} x_{2} ; x_{1}{ }^{\prime} x_{2}{ }^{\prime}\right) \varphi\left(x_{1}{ }^{\prime} x_{2}{ }^{\prime}\right) \mathrm{d} x_{1}{ }^{\prime} \mathrm{d} x_{2}{ }^{\prime}
\end{aligned}
$$

erhält man nach 1 a), indem man Abb. 5 durch $S$-Linien ergänzt und über alle möglichen Graphen summiert. Diese Graphen zerfallen in zwei Gruppen. Bei der ersten gibt es keine $S$-Linie, die die linke Hälfte des Bildes mit der rechten verbindet. (Beispiel: Abb. 6.) Bei der zweiten gibt es solche Verbindungslinien (Beispiel: Abb. 7).

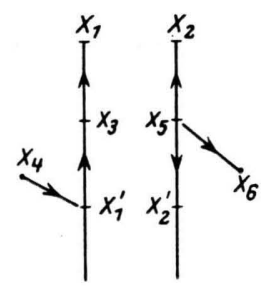

Abb. 5.

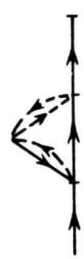

Abb. 6.

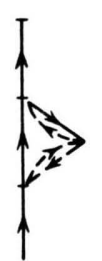

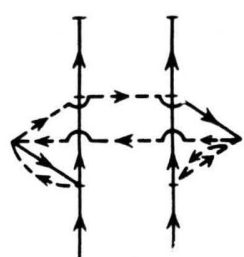

Abb. 7.
Den Teil des Kerns $K$, der von allen Graphen der ersten Gruppe herrührt, bezeichnen wir mit $K_{\mathrm{s}}$, den zweiten Teil mit $K_{\mathrm{w}}$.

$$
K=K_{\mathrm{s}}+K_{\mathrm{w}} .
$$

(Die Indizes s und w sollen an den Zusammenhang mit der Selbstenergie bzw. der Wechselwirkung erinnern.)

Ferner versuchen wir eine Entwicklung der Wellenfunktion

$$
\varphi=\varphi_{0}+\varphi_{1}+\varphi_{2}+\ldots
$$


wobei die Funktion $\varphi_{0}$ die einlaufenden Wellen, d. h. einfach ein Produkt von zwei ebenen Wellen mit den richtigen Ruhmassen (genauer: im Beispiel mit den Ruhmassen, die in der Näherung $N=5$ für das einzelne Teilchen berechnet werden) bedeuten soll. Aus der Integralgleichung (9) folgt:

$$
\begin{aligned}
\varphi_{0}+\varphi_{1}+\ldots=K_{\mathrm{s}}\left(\varphi_{0}\right. & \left.+\varphi_{1}+\ldots\right) \\
& +K_{\mathrm{w}}\left(\varphi_{0}+\varphi_{1}+\ldots\right) .
\end{aligned}
$$

Da nun nach der Berechnung der Ruhmassen

gilt, so ergibt sich $\varphi_{0}=K_{\mathrm{s}} \varphi_{0}$

$$
\left(1-K_{\mathrm{s}}\right) \varphi_{1}=K_{\mathrm{w}} \varphi_{0}
$$

und durch rein formale Integration allgemein

$\varphi_{n}=\left(1-K_{\mathrm{s}}\right)^{-1} K_{\mathrm{w}} \varphi_{n-1}=\left[\left(1-K_{\mathrm{s}}\right)^{-1} K_{\mathrm{w}}\right]^{n} \varphi_{0}$.

Bricht man mit $\varphi_{1}$ ab, so entspricht dies der Bornschen Näherung.

Es muß nun noch untersucht werden, wie der formale Operator $\left(1-K_{\mathrm{s}}\right)^{-1} K_{\mathrm{w}}$ unter Berücksichtigung der Randbedingungen ausgewertet werden kann. $\mathrm{Zu}$ diesem $\mathrm{Z}$ weck wendet man auf die beiden Seiten der Gl.(13) den Dirac-Operator $L=\gamma_{\mu} \partial / \partial x_{\mu}$ sowohl für die Variable $x_{1}$ als auch für $x_{2}$ an. Auf der rechten Seite werden dabei die Greenschen Funktionen $G_{\mathrm{F}}\left(x_{1}, x_{3}\right)$ bzw. $G_{\mathrm{F}}\left(x_{2}, x_{5}\right)$ durch die entsprechenden $\delta$-Funktionen ersetzt. Auf der linken Seite entsteht der Ausdruck

$$
L_{1} L_{2} \varphi_{1}-L_{1} L_{2} K_{\mathrm{s}} \varphi_{1},
$$

der dann verschwindet, wenn $\varphi_{1}$ ein Produkt von ebenen Wellen der richtigen Massen oder eine Summe von solchen Produkten ist. Es liegt daher nahe, den Ausdruck (15) in folgender Weise umzuschreiben $(\varkappa$ sei die Ruhmasse, die sich in der Näherung $N=5$ ergibt):

$$
\begin{array}{r}
L_{1} L_{2} \varphi_{1}-L_{1} L_{2} K_{\mathrm{s}} \varphi_{1}=\left(L_{1}+\varkappa\right)\left(L_{2}+\varkappa\right) \varphi_{1} \\
-\left[\varkappa\left(L_{2}+\varkappa\right)+\varkappa\left(L_{1}+\varkappa\right)+L_{1} L_{2} K_{\mathrm{s}}-\varkappa^{2}\right] \varphi_{1}
\end{array}
$$

Dann kann man das Gleichungssystem (13) und (14) durch das folgende ersetzen:

$$
\begin{array}{r}
\left(L_{1}+\varkappa\right)\left(L_{2}+\varkappa\right) \varphi_{1}=L_{1} L_{2} K_{\mathrm{w}} \varphi_{0} \quad \text { und } \quad(17) \\
\left(L_{1}+\varkappa\right)\left(L_{2}+\varkappa\right) \varphi_{n}=\left[L_{1} L_{2} K_{\mathrm{w}}+\varkappa\left(L_{2}+\varkappa\right)\right. \\
\left.+\varkappa\left(L_{1}+\varkappa\right)+L_{1} L_{2} K_{\mathrm{s}}-\varkappa^{2}\right] \varphi_{n-1} \text { für } n>1 .
\end{array}
$$

Integriert man diese Gleichungen mit Hilfe der Greenschen Funktionen zur Masse $\varkappa$, so wird in erster Näherung

$$
\varphi_{1}=K_{\mathrm{W}}^{\mathrm{r}} \varphi_{0},
$$

wobei $\operatorname{sich} K_{\mathrm{W}}^{\mathrm{r}}$ (sozusagen der renormierte Operator $K_{\mathrm{W}}$ ) von $K_{\mathrm{W}}$ nur dadurch unterscheidet, daß für die letzten, zu den Endpunkten führenden $G$ Linien nicht die masselose $G_{\mathrm{F}}$-Funktion, sondern die zur Masse $*$ gehörige $G_{\mathrm{F}}^{\varkappa}$-Funktion einzusetzen ist.

Die Gleichungen höherer Ordnungen werden etwas komplizierter durch die Korrekturglieder auf der rechten Seite von (18), es bleibt aber offenbar $K_{\mathrm{W}}^{\mathrm{r}}$ der wesentliche Teil des Wechselwirkungsoperators.

Wenn man die Gln. (13) und (14) oder (17) und (18) formal bis zu beliebig hoher Näherung integriert und $\varphi=\Sigma \varphi_{n}$ aufsummiert, so kommt man, wie man leicht nachrechnet, einfach zur homogenen Integralgleichung $\varphi=K \varphi$ zurück.

Die Auswahl des $G$-Linienbildes, von dem man ausgeht, ist auch hier bis zu einem gewissen Grade willkürlich. $\mathrm{Zu}$ den Regeln von 1 a) kommt hier nur die zusätzliche Einschränkung, daß ein $G$ Linienzug von $x_{1}^{\prime}$ nach $x_{1}$, ein anderer von $x_{2}^{\prime}$ nach $x_{2}$ führen soll. Es gibt aber noch viele $G$-Linienbilder, die dieser Bedingung genügen, und es wird daher im Hinblick auf die Bethe-Salpeter-Gleichung der üblichen Theorie wieder zweckmäßig sein, über die entsprechenden Integralgleichungen zu mitteln, um die beste Approximation zu erhalten. Es muß aber noch einmal hervorgehoben werden, daß die ganzen hier besprochenen Integralgleichungssysteme erheblich ungenauer sind als die in I und II zugrunde gelegten $\tau$ - bzw. $\varphi$ Gleichungssysteme; nur sind die letzteren in den meisten Fällen für die Ausrechnung zu kompliziert.

Aus dem geschilderten Rechnungsgang erkennt man, daß als einlaufende oder auslaufende Wellen nur Produkte von ebenen Wellen für wirkliche Teilchen, d. h. Lösungen der Integralgleichung $\varphi=K_{\mathrm{S}} \varphi$ vorkommen. $\mathrm{Zu}$ diesen ,,wirklichen Teilchen“ gehören natürlich auch alle gebundenen Zustände, die im Graphenschema als periodische Leitergraphen oder ,ZZ̈pfe“ (oder als im Ring geschlossene Linienmuster) dargestellt werden. Durch eine geringfügige Erweiterung der eben dargestellten Rechnungen kann man die Fälle einschließen, in denen beim Stoß neue Teilchen erzeugt oder vorhandene vernichtet werden. In dieser Weise erhält man die vollständige $S$-Matrix zu der betreffenden Energie.

Diese $S$-Matrix ist unitär, wie in der folgenden Weise begründet werden kann. Die Gesamtheit der zur gegebenen Energie gehörigen Zustände des 
Hilbert-Raums I kann erstens charakterisiert werden durch ihr Verhalten bei $t=-\infty$, d. h. durch ihre einlaufenden Wellen. Wir nennen die so charakterisierten Zustände $\Phi_{\text {ein }}$; zweitens kann zur Charakterisierung das Verhalten bei $t=+\infty$, d. h. die auslaufenden Wellen verwendet werden, die so bezeichneten Zustände sollen $\Phi_{\text {aus }}$ heißen. Die Zustände sind zeitunabhängig, ihre darstellenden $\varphi$ oder $\tau$-Funktionen genügen dem gleichen linearen Integral- oder Differentialgleichungssystem. Die Gesamtheit der Zustände $\Phi_{\text {ein }}$ spannt also den gleichen Unterraum des Hilbert-Raums I auf wie die Gesamtheit der Zustände $\Phi_{\text {aus }}$, nämlich den vollen zur gegebenen Energie gehörigen Unterraum. Da die Metrik im Hilbert-Raum I jedenfalls positiv definit ist - denn so ist der Hilbert-Raum I definiert -, muß die lineare Transformationsmatrix, die von einem vollständigen Orthogonalsystem der $\Phi_{\text {ein }}$ zu einem vollständigen Orthogonalsystem der $\Phi_{\text {aus }}$ führt, nach allgemeinen Sätzen unitär sein. Die Matrixelemente der $S$-Matrix sind wie in der üblichen Theorie die skalaren Produkte $\left(\Phi_{\text {ein }}, \Phi_{\text {aus }}\right)$.

Die eben angeführte Überlegung beweist zunächst noch nicht, daß die Normierung der $\mathrm{Zu}$ stände, wenn sie etwa mit Hilfe der Amplitude der ebenen Wellen bei $t=-\infty$ vorgenommen wird, die gleiche ist, wie wenn sie mit den ebenen Wellen bei $t=+\infty$ durchgeführt wird. Die Gleichheit der Normierung geht erst aus der Symmetrie des mathematischen Formalismus gegenüber der Vorzeichenumkehr der Zeit hervor, die sich in der Benutzung der Funktionen $S_{\mathrm{F}}$ und $G_{\mathrm{F}}$ ausspricht. Außerdem setzt das ganze Schlußverfahren voraus, daß die geschilderte Näherungsrechnung mit wachsendem $N$ gegen einen Grenzwert konvergiert, was bisher nicht bewiesen ist.

\section{c) Spinorteilchen}

Nun soll die Masse des leichtesten Spinorteilchens nach den besprochenen Verfahren genauer als in II $^{5}$ berechnet werden. $\mathrm{Zu}$ diesem Zweck betrachten wir das Integralgleichungssystem für die $\tau$-Funktionen zur ,Ladung" eins in der Näherung $N=3$ :

$$
\begin{gathered}
\varphi_{\alpha}(x \mid)=\tau_{\alpha}(x \mid)=-l^{2} i / 2 \int G_{\alpha \mu}\left(x-x^{\prime}\right) \\
\cdot \tau_{\mu \varrho / \varrho}\left(x^{\prime} x^{\prime} \mid x^{\prime}\right) \mathrm{d}^{4} x^{\prime} \\
\tau_{\alpha \beta \gamma}(x y \mid z) \quad-l^{2} i / 2 \int G_{\alpha \mu}\left(x-x^{\prime}\right) \\
=-\tau_{\mu \beta \varrho / \varrho \gamma}\left(x^{\prime} y x^{\prime} \mid x^{\prime} z\right) \mathrm{d}^{4} x^{\prime} \\
\quad \cdot 2 \int G_{\beta \nu}\left(y-y^{\prime}\right) \\
=-\tau_{\alpha \nu \sigma / \sigma \gamma}\left(x y^{\prime} y^{\prime} \mid y^{\prime} z\right) \mathrm{d}^{4} y^{\prime} \\
\cdot \tau_{\alpha \beta \tau / \tau \varrho}\left(x y z^{\prime} \mid z^{\prime} z^{\prime}\right) \mathrm{d}^{4} z^{\prime} .
\end{gathered}
$$

Mitteln wir über die drei Gleichungen für $\tau(x y \mid z)$ und setzen in die erste Gleichung ein, so erhalten wir

$$
\begin{aligned}
& \varphi_{\alpha}(x \mid)=\tau_{\alpha}(x \mid)=-\frac{l^{4}}{12} \int_{\alpha \mu}\left(x-x^{\prime}\right) \\
& \cdot\left[G_{\mu \sigma}\left(x^{\prime}-x^{\prime \prime}\right) \tau_{\sigma \varrho \delta / \delta \varrho}\left(x^{\prime \prime} x^{\prime} x^{\prime \prime} \mid x^{\prime \prime} x^{\prime}\right)\right. \\
& +G_{\varrho \nu}\left(x^{\prime}-x^{\prime \prime}\right) \tau_{\mu \nu \sigma / \sigma \varrho}\left(x^{\prime} x^{\prime \prime} x^{\prime \prime} \mid x^{\prime \prime} x^{\prime}\right) \\
& \left.+G_{\varepsilon \varrho}\left(x^{\prime \prime}-x^{\prime}\right) \tau_{\mu \varrho \tau / \tau \varepsilon}\left(x^{\prime} x^{\prime} x^{\prime \prime} \mid x^{\prime \prime} x^{\prime \prime}\right)\right] \mathrm{d}^{4} x^{\prime} \mathrm{d}^{4} x^{\prime \prime} .
\end{aligned}
$$

Nun müssen die $\tau$-Funktionen durch $\varphi$-Funktionen und Kontraktionen ausgedrückt werden. In der so entstehenden Integralgleichung werden alle $\varphi$-Funktionen bis auf die niedrigste weggelassen. Wir erhalten

$$
\begin{aligned}
& \text { Die zugehörigen Graphen sind von dem } \\
& \text { Typus 8. Nun wenden wir auf beiden Seiten } \\
& \text { den Operator } \gamma_{\mu} \partial / \partial x_{\mu} \text { an und machen von } \\
& \text { der Tatsache Gebrauch, daß } \varphi \text { sich auf einen }
\end{aligned}
$$$$
\varphi_{\alpha}(x \mid)=-\frac{l^{4}}{48} \int G_{\alpha \mu}\left(x-x^{\prime}\right)\left[G_{\mu \sigma}\left(x^{\prime}-x^{\prime \prime}\right) S_{\varrho \delta}\left(x^{\prime}-x^{\prime \prime}\right) S_{\varrho \sigma}\left(x^{\prime \prime}-x^{\prime}\right) \varphi_{\delta}\left(x^{\prime \prime} \mid\right)\right.
$$$$
-G_{\mu \sigma}\left(x^{\prime}-x^{\prime \prime}\right) S_{\varrho \delta}\left(x^{\prime}-x^{\prime \prime}\right) S_{\delta \varrho}\left(x^{\prime \prime}-x^{\prime}\right) \varphi_{\sigma}\left(x^{\prime \prime} \mid\right)+G_{\varrho \nu}\left(x^{\prime}-x^{\prime \prime}\right) S_{\mu \sigma}\left(x^{\prime}-x^{\prime \prime}\right) S_{\sigma \varrho}\left(x^{\prime \prime}-x^{\prime}\right) \varphi_{\nu}\left(x^{\prime \prime} \mid\right)
$$$$
-G_{\varrho \nu}\left(x^{\prime}-x^{\prime \prime}\right) S_{\mu \sigma}\left(x^{\prime}-x^{\prime \prime}\right) S_{\nu \varrho}\left(x^{\prime \prime}-x^{\prime}\right) \varphi_{\sigma}\left(x^{\prime \prime} \mid\right)+G_{\varepsilon \varrho}\left(x^{\prime \prime}-x^{\prime}\right) S_{\varrho \tau}\left(x^{\prime}-x^{\prime \prime}\right) S_{\mu \varepsilon}\left(x^{\prime}-x^{\prime \prime}\right) \varphi_{\tau}\left(x^{\prime \prime} \mid\right)
$$$$
\left.-G_{\varepsilon \varrho}\left(x^{\prime \prime}-x^{\prime}\right) S_{\varrho \varepsilon}\left(x^{\prime}-x^{\prime \prime}\right) S_{\mu \tau}\left(x^{\prime}-x^{\prime \prime}\right) \varphi_{\tau}\left(x^{\prime \prime} \mid\right)\right] \mathrm{d}^{4} x^{\prime} \mathrm{d}^{4} x^{\prime \prime} \text {. }
$$

Abb. 8. Zustand zum Viererimpuls $J_{\mu}$ beziehen soll.
Dann gilt

$$
\left(\gamma_{\mu}\right)_{\alpha \beta} \frac{\partial}{\partial x_{\mu}} \varphi_{\beta}(x \mid)=-\underline{J}_{\alpha \beta} \varphi_{\beta},
$$

wobei $\underline{A}=-i \gamma_{\nu} A_{\nu}$ bedeuten soll. Außerdem ist

$$
\left(\gamma_{\mu}\right)_{\alpha \nu} \frac{\partial}{\partial x_{\mu}} G_{\alpha \beta}\left(x-x^{\prime}\right)=2 i \delta\left(x-x^{\prime}\right) \delta_{\nu^{\prime} \beta} .
$$

Damit erhält die Eigenwertgleichung die Form

$$
\begin{aligned}
& \underline{J \mu \alpha}_{\mu \alpha}(x \mid)=+\frac{i l^{4}}{24} \int \mathrm{d}^{4} x^{\prime}\left[G_{\mu \sigma}\left(x-x^{\prime}\right) S_{\varrho \delta}\left(x-x^{\prime}\right) S_{\sigma \varrho}\left(x^{\prime}-x\right) \varphi_{\delta}\left(x^{\prime} \mid\right)-G_{\mu \sigma}\left(x-x^{\prime}\right) S_{\varrho \delta}\left(x-x^{\prime}\right) S_{\delta \varrho}\left(x^{\prime}-x\right) \varphi_{\sigma}\left(x^{\prime} \mid\right)\right. \\
& +G_{\varrho \nu}\left(x-x^{\prime}\right) S_{\mu \sigma}\left(x-x^{\prime}\right) S_{\sigma \varrho}\left(x^{\prime}-x\right) \varphi_{\nu}\left(x^{\prime} \mid\right)-G_{\varrho \nu}\left(x-x^{\prime}\right) S_{\mu \sigma}\left(x-x^{\prime}\right) S_{\nu \varrho}\left(x^{\prime}-x\right) \varphi_{\sigma}\left(x^{\prime} \mid\right) \\
& \left.+G_{\varepsilon \varrho}\left(x^{\prime}-x\right) S_{\varrho \tau}\left(x-x^{\prime}\right) S_{\mu \varepsilon}\left(x-x^{\prime}\right) \varphi_{\tau}\left(x^{\prime} \mid\right)-G_{\varepsilon \varrho}\left(x^{\prime}-x\right) S_{\varrho \varepsilon}\left(x-x^{\prime}\right) S_{\mu \tau}\left(x-x^{\prime}\right) \varphi_{\tau}\left(x^{\prime} \mid\right)\right] .
\end{aligned}
$$

${ }^{5}$ Die Rechnung in II enthielt einige Vorzeichenfehler und andere Ungenauigkeiten, die sich aber im Ergebnis nur wenig auswirken. 
Die weitere Rechnung wird im Impulsraum durchgeführt, weil das einfacher und zweckmäßiger ist. Wir setzen

$\varphi_{\alpha}(x \mid)=e^{i . J_{\mu} x_{\mu}} \varphi_{\alpha}$,

$G(x)=\frac{1}{(2 \pi)^{4}} \int e^{i p_{\mu} x_{\mu}} G(p)$, ebenso für $S$,

und finden nach einigen einfachen Umformungen

$\underline{J}_{\alpha \xi} \varphi_{\xi}=+\frac{i l^{4}}{24(2 \pi)^{8}} \int \mathrm{d}^{4} q \mathrm{~d}^{4} r$

$$
\begin{gathered}
\cdot\left[G_{\alpha \mu}(q-r+J) S_{\nu \beta}(q) S_{\beta \nu}(r)\right. \\
-G_{\alpha \nu}(q-r+J) S_{\nu \beta}(q) S_{\beta \mu}(r) \\
-G_{\beta \mu}(q-r+J) S_{\nu \beta}(q) S_{\alpha \nu}(r) \\
+G_{\beta \nu}(q-r+J) S_{\nu \beta}(q) S_{\alpha \mu}(r) \\
+G_{\nu \beta}(q-r+J) S_{\alpha \nu}(-q) S_{\beta \mu}(r) \\
\left.-G_{\nu \beta}(q-r+J) S_{\alpha \mu}(-q) S_{\beta \nu}(r)\right] \varphi_{\mu} .
\end{gathered}
$$

In diese Formel werden nun die Impulsdarstellungen der Funktionen $S$ und $G$ eingesetzt:

$$
\begin{aligned}
G(p)= & \lim _{\delta \rightarrow 0} 2 i \frac{\underline{p}}{p^{2}-i \delta}, \\
S(p)= & \lim _{\delta \rightarrow 0} 2 i\left[\frac{\underline{p} \varkappa^{2}}{\left(p^{2}-i \delta\right)^{2}\left(p^{2}+\varkappa^{2}-i \delta\right)}\right. \\
& \left.\quad-\frac{\varkappa^{3}}{\left(p^{2}-i \delta\right)\left(p^{2}+\varkappa^{2}-i \delta\right)}\right] .
\end{aligned}
$$

Der entstehende lange Ausdruck kann wesentlich vereinfacht werden, indem in einigen Gliedern $p$ durch $-q$ und $q$ durch $-p$ ersetzt wird. Ein Teil der Matrizenprodukte kann ebenfalls ausgeführt werden, wir erhalten nach einiger Rechnung

$$
\begin{aligned}
\underline{J}_{\alpha \xi} \varphi_{\xi}= & +\frac{l^{4} \varkappa^{6}}{3(2 \pi)^{8}} \int_{\delta \rightarrow 0} \mathrm{~d}^{4} q \mathrm{~d}^{4} r \frac{1}{\left[(q-r+J)^{2}-i \delta\right]\left[q^{2}-i \delta\right]\left[q^{2}+\varkappa^{2}-i \delta\right]\left[r^{2}-i \delta\right]\left[r^{2}+\varkappa^{2}-i \delta\right]} \cdot\{3 \underline{(q}-\underline{r}+\underline{J})_{\alpha \xi} \varphi_{\xi} \\
& \left.+6 \varkappa \frac{(q, q-r+J)}{q^{2}-i \delta} \varphi_{\alpha}-\frac{x^{2}}{\left(q^{2}-i \delta\right)\left(r^{2}-i \delta\right)}[3(q r)(\underline{q}-\underline{r}+\underline{J})+6(q, q-r+J) \underline{r}]_{\alpha \xi} \varphi_{\xi}\right\} .
\end{aligned}
$$

Nun müssen die Integrale ausgewertet werden. Es ist am besten, das allgemeinere Integral

$$
K=\int \mathrm{d}^{4} q \mathrm{~d}^{4} r \frac{\underline{q-} \underline{r}+\underline{J}}{\left[(q-r+J)^{2}-i \delta\right]\left[q^{2}+(a q)-i \delta\right]\left[r^{2}-(b r)-i \delta\right]\left[q^{2}+\varkappa^{2}-i \delta\right]\left[r^{2}+\varkappa^{2}-i \delta\right]}
$$

zu berechnen. Mit seiner Hilfe lautet die Eigenwertgleichung

$$
J \varphi=+\frac{l^{4} \varkappa^{6}}{3(2 \pi)^{8}}\left\{3 K_{\mid a=b=0}-\left.\frac{3 \varkappa}{2} i \operatorname{Sp}\left(\gamma_{\nu} \frac{\partial K}{\partial a_{\nu}}\right)\right|_{a=b=0}+3 \varkappa^{2}\left[\frac{\partial^{2} K}{\partial a_{\mu} \partial b_{\mu}}+\frac{1}{2} \operatorname{Sp}\left(\gamma_{\mu} \frac{\partial^{2} K}{\partial a_{\mu} \partial b_{\nu}}\right) \gamma_{\nu}\right]_{a=b=0}\right\} .
$$

Die Berechnung von $K$ erfolgt mit Hilfe der Feynmanschen Methoden mit Verwendung der Formel

$$
\frac{1}{a b}=\int_{0}^{1} \frac{\mathrm{d} x}{[a x+b(1-x)]^{2}}
$$

und ähnlicher, die daraus durch Differenzieren zu erhalten sind. Dadurch wird die Zahl der Faktoren im Nenner herabgesetzt, und die Integration über die Impulsvariabeln ist mit Hilfe der Resultate von Feynman (Phys. Rev. 76, 769 [1949] Appendix) leicht möglich. Für $K$ und die daraus durch Differenzieren gewonnenen Ausdrücke, die in die Gleichung zur Bestimmung des Eigenwertes eingesetzt werden müssen, erhält man mehrfache Integrale über die Hilfsvariabeln, die von der obigen Zerlegung herrühren. Die Berechnung dieser Integrale ist analytisch, wenn überhaupt möglich, sehr kompliziert, so daß die Auswertung mit der elektronischen Rechenmaschine G 1 des Max-Planck-Instituts für Physik vorgezogen wurde. Die Eigenwertgleichung erhält schließlich die Form

$$
\frac{1}{\varkappa} \underline{J}=\left(\frac{l \varkappa}{4 \pi}\right)^{4}\left[\frac{1}{\varkappa} \underline{J} Q\left(X^{2}\right)+R\left(X^{2}\right)\right]
$$

wobei $X^{2}=-J^{2} / \varkappa^{2}$ ist. $X^{2}$ ist stets positiv.

Die Größen $Q\left(X^{2}\right)$ und $R\left(X^{2}\right)$ bestehen im wesentlichen aus dem ersten und dritten bzw. aus dem zweiten Glied der rechten Seite von (30). Beide Funktionen wurden für verschiedene Werte von $X^{2}$ zwischen 0 und 7 numerisch ermittelt. Die Resultate sind

\begin{tabular}{|l|l|l|l|l|l|}
\hline$X^{2}=$ & 0,0 & 0,2 & 0,4 & 0,6 & 0,8 \\
$Q=$ & & 0,879 & 2,243 & 3,019 & 3,507 \\
$R=$ & 4,290 & 4,692 & 4,930 & 4,849 & 4,767 \\
\hline$X^{2}=$ & 1,0 & 2,0 & 3,0 & 5,0 & 7,0 \\
$Q=$ & 3,780 & 3,388 & 2,427 & 1,285 & 0,914 \\
$R=$ & 4,612 & 3,209 & 1,958 & 0,543 & 0,261 \\
\hline
\end{tabular}

Wenn der betrachtete Zustand den Impuls Null hat, ist $X^{2}=x_{n}^{2} / \varkappa^{2}$ und $\underline{J} / \varkappa=\underline{X}=\beta \varkappa_{n} / \varkappa$. Es bleibt die Gleichung

$$
\frac{\varkappa_{n}}{\varkappa}=\left(\frac{l \varkappa}{4 \pi}\right)^{4}\left[\frac{\varkappa_{n}}{\varkappa} Q_{ \pm} R\right] .
$$

Für den tiefsten Eigenwert soll $\varkappa_{n}=\varkappa$, d. h. $X=1$ sein. Man erhält daher aus (32 a)

$$
\varkappa l=7,426
$$

für die Masse des leichtesten Spinorteilchens. (Die frühere ungenaue Rechnung in l. c. II hatte 7,45, also zufällig fast das gleiche Ergebnis geliefert.) Andere Eigenwerte gibt es in dieser Näherung nicht, wie einzusehen ist, wenn man die linke und rechte Seite von (32a) als Funktion von $X$ graphisch darstellt (Abb. 9 $F_{1}$ stellt die linke, $F_{2}$ bzw. $F_{3}$ die rechte Seite mit positivem bzw. negativen Vorzeichen dar). 


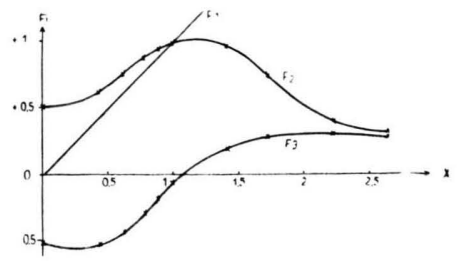

Abb. 9.

\section{Wechselwirkung und gebundene Zustände}

a) Kraft zwischen zwei Spinorteilchen

Der Charakter der Kraft zwischen zwei Spinorteilchen kann aus der gegenseitigen Streuung dieser Teilchen ersehen werden. Die Übergangsamplitude für die Streuung ist nach dem unter 1 b) angegebenen Verfahren zu ermitteln. Dabei werden sich die Kräfte langer und kurzer Reichweite verschieden in der Streuung auswirken. Für große Änderungen der Teilchenimpulse $\left[\left(p_{\lambda}-p_{\lambda}{ }^{\prime}\right)^{2} \sim \chi^{2}\right]$ werden die Kräfte kurzer Reichweite mindestens ebensoviel zur Streuamplitude beitragen wie die Kräfte langer Reichweite. Für sehr kleine Änderungen $\left[\left(p_{\lambda}-p_{\lambda}{ }^{\prime}\right)^{2} \ll \varkappa^{2}\right]$ dagegen rührt der entscheidende Beitrag von den Kräften langer Reichweite her, sofern solche vorhanden sind; die Streuamplitude wird um so größer, je kleiner die Impulsdifferenz ist. In den folgenden Rechnungen werden wir uns in erster Linie für die Kräfte langer Reichweite interessieren, also für den Streuquerschnitt bei sehr kleinen Impulsdifferenzen. Nur in diesem Grenzfall soll die Berechnung vollständig durchgeführt werden.

Wir leiten zunächst im Anschluß an 1 b) die Übergangsamplitude allgemein ab. Sei $\varphi_{\alpha \beta}^{\prime(0)}(x, y \mid)$ die Wellenfunktion von zwei freien Teilchen der Masse $\varkappa$, so gilt

$$
\begin{array}{r}
\left(\gamma_{\nu} \frac{\partial}{\partial x_{\nu}}+\chi\right)_{\mu \alpha} \varphi_{\alpha \beta}^{\prime(0)}(x, y \mid)= \\
{ }^{\prime} \gamma_{\alpha \beta}^{\prime(0)}(x, y \mid)=0,
\end{array}
$$

und $\varphi_{\alpha \beta}^{\prime(0)}(x, y \mid)$ hat die Form

$$
\begin{array}{r}
\varphi_{\alpha \beta}^{\prime(0)}(x, y \mid)=u_{\alpha \beta}\left(p^{\prime}, q^{\prime} \mid\right) e^{i\left(p^{\prime} x\right)} e^{i\left(q^{\prime} y\right)}, \\
p^{\prime 2}=q^{\prime 2}=-\varkappa^{2} .
\end{array}
$$

Die Übergangsamplitude ist dann gegeben durch

$A=\iint \mathrm{d}^{3} x \mathrm{~d}^{3} y \bar{\varphi}_{\alpha \beta}^{\prime(0)}(x, y \mid) \beta_{\alpha \mu} \beta_{\beta \nu} \varphi_{\mu \nu}^{\text {aus }}(x, y \mid)$

[vgl. z. B. Freese*].

* E. Freese, Z. Naturforschg. 8 a, 776 [1953].
Wenn die Wellenfunktion der einlaufenden Teilchen zu der der auslaufenden Teilchen orthogonal ist (wenn also die auslaufenden Teilchen andere Impulse haben), kann dieser Ausdruck in ein Integral über den ganzen vierdimensionalen Raum umgeformt werden. Mit Verwendung der Dirac-Gleichung für $\varphi$ und des Gaußschen Satzes erhält man

$$
\begin{gathered}
A=-\iint \mathrm{d}^{4} x \mathrm{~d}^{4} y \bar{\varphi}_{\mu \nu}^{\prime(0)}(x, y \mid)\left(\gamma_{\varrho} \frac{\partial}{\partial x_{\varrho}}+\chi\right)_{\mu \alpha} \\
\cdot\left(\gamma_{\sigma} \frac{\partial}{\partial y_{\sigma}}+\chi\right)_{\nu \beta} \varphi_{\alpha \beta}(x, y \mid) .
\end{gathered}
$$

$\operatorname{Nach}(17)$ ist nun $\left(\gamma_{\nu} \partial / \partial x_{\nu}+\varkappa\right)_{\mu \alpha}\left(\gamma_{\mu} \partial / \partial x_{\mu}+\varkappa\right)_{\nu \beta} \varphi_{\alpha \beta}(x, y \mid)$ in niedrigster Näherung leicht zu berechnen, wenn der die Wechselwirkung vermittelnde Anteil des Integralkernes aus der Integralgleichung für $\varphi(x y \mid)$ bekannt ist, wobei diese Integralgleichung nach dem vorne geschilderten Verfahren gefunden wird. Aus den Regeln des Abschnittes über Streuprobleme folgt, daß dieser stets die Form

$$
\begin{aligned}
K_{\mathrm{w} \alpha \beta \mid \gamma \delta}\left(x y \mid x^{\prime} y^{\prime}\right)=- & \frac{l^{4}}{4} \iint \mathrm{d}^{4} x^{\prime \prime} \mathrm{d}^{4} y^{\prime \prime} G_{\alpha \mu \mu}\left(x-x^{\prime \prime}\right) \quad(37) \\
& \cdot G_{\beta \nu}\left(y-y^{\prime \prime}\right) N_{\mu \nu \mid \gamma \delta}\left(x^{\prime \prime} y^{\prime \prime} \mid x^{\prime} y^{\prime}\right)
\end{aligned}
$$

haben muß. Nun ist [vgl. (17)]

$$
\begin{aligned}
& \left(\gamma_{\varrho} \frac{\partial}{\partial x_{\varrho}}+x\right)_{\mu \alpha}\left(\gamma_{\sigma} \frac{\partial}{\partial x_{\sigma}}+\chi\right)_{\nu \beta} \varphi_{\alpha \beta}^{(x, y)} \\
& =\left(\gamma_{\varrho} \frac{\partial}{\partial x_{\varrho}}\right)_{\mu \alpha}\left(\gamma_{\sigma} \frac{\partial}{\partial x_{\sigma}}\right)_{\nu \beta} \iint \mathrm{d}^{4} x^{\prime} \mathrm{d}^{4} y^{\prime} K_{\mathrm{W} \alpha \beta \mid \gamma \delta}\left(x y \mid x^{\prime} y^{\prime}\right) \\
& \cdot \varphi_{\gamma \delta}^{(0)}\left(x^{\prime}, y^{\prime} \mid\right)=+l^{4} \iint \mathrm{d}^{4} x^{\prime} \mathrm{d}^{4} y^{\prime} N_{\mu \nu} / \gamma \delta \\
& \left(x y \mid x^{\prime} y^{\prime}\right) \varphi_{\gamma \delta}^{(0)}\left(x^{\prime}, y^{\prime} \mid\right) ;
\end{aligned}
$$

also hat die Übergangsamplitude die Form

$$
\begin{array}{r}
A=-l^{4} \iint \mathrm{d}^{4} x \mathrm{~d}^{4} y \iint \mathrm{d}^{4} x^{\prime} \mathrm{d}^{4} y^{\prime} \bar{\varphi}_{\mu \nu}^{(0)}(x y \mid) N_{\mu \nu \mid \gamma \delta}\left(x y \mid x^{\prime} y^{\prime}\right) \\
\varphi_{\gamma \delta}^{(0)}\left(x^{\prime}, y^{\prime} \mid\right) .
\end{array}
$$

Nun muß der Kern $N$ ermittelt werden. Dazu betrachten wir das Integralgleichungssystem $\operatorname{der} \tau$-Funktionen der ,Ladung“" zwei:

$$
\begin{aligned}
\tau_{\alpha \beta}(x, y \mid)=- & \frac{i l^{2}}{2} \int G_{\alpha \mu}\left(x-x^{\prime}\right) \\
& \cdot \tau_{\mu \beta \varrho / \varrho}\left(x^{\prime} y x^{\prime} \mid x^{\prime}\right) \mathrm{d}^{4} x^{\prime}, \\
\tau_{\alpha \beta \gamma / \delta}(x y z \mid w)=- & \frac{i l^{2}}{2} \int G_{\beta \nu}\left(y-y^{\prime}\right) \\
& \cdot \tau_{\alpha \nu \gamma \varrho / \varrho \delta}\left(x y^{\prime} z y^{\prime} \mid y^{\prime} w\right) \mathrm{d}^{4} y^{\prime} .
\end{aligned}
$$

Durch Einsetzen der zweiten Gleichung in die erste erhalten wir

$$
\begin{array}{r}
\tau_{\alpha \beta}(x, y \mid)=-\frac{l^{4}}{4} \iint G_{\alpha, u}\left(x-x^{\prime}\right) G_{\beta \nu}\left(y-y^{\prime}\right) \\
\cdot \tau_{\mu \nu \varrho \sigma / \sigma \varrho}\left(x^{\prime} y^{\prime} x^{\prime} y^{\prime} \mid y^{\prime} x^{\prime}\right) \mathrm{d}^{4} x^{\prime} \mathrm{d}^{4} y^{\prime} .
\end{array}
$$

Eine Mittelung ist wegen der symmetrischen Form der Gleichung nicht nötig. Setzt man für die $\tau$-Funktionen die $\varphi$-Funktionen ein und behält nur die niedrigste $\varphi$ Funktion, so erhält man 


$$
\begin{gathered}
\varphi_{\alpha \beta}(x, y \mid)=-\frac{l^{4}}{16} \int \mathrm{d}^{4} x^{\prime} \int \mathrm{d}^{4} y^{\prime} G_{\alpha \mu}\left(x-x^{\prime}\right) G_{\beta \nu}\left(y-y^{\prime}\right) \\
\cdot\left[S_{\mu \sigma}\left(x^{\prime}-y^{\prime}\right) S_{\sigma \varrho}\left(y^{\prime}-x^{\prime}\right) \varphi_{\varrho \nu}^{(0)}\left(x^{\prime}, y^{\prime} \mid\right)\right. \\
-S_{\varrho \sigma}\left(x^{\prime}-y^{\prime}\right) S_{\sigma \varrho}\left(y^{\prime}-x^{\prime}\right) \varphi_{\mu \nu}^{(0)}\left(x^{\prime}, y^{\prime} \mid\right) \\
+S_{\varrho \sigma}\left(x^{\prime}-y^{\prime}\right) S_{\nu \varrho}\left(y^{\prime}-x^{\prime}\right) \varphi_{\mu \sigma}^{(0)}\left(x^{\prime}, y^{\prime} \mid\right) \\
\left.-S_{\mu \sigma}\left(x^{\prime}-y^{\prime}\right) S_{\nu \varrho}\left(y^{\prime}-x^{\prime}\right) \varphi_{\varrho \sigma}^{(0)}\left(x^{\prime}, y^{\prime} \mid\right)\right] .
\end{gathered}
$$

Damit ist der Kern in der Näherung $N=4$ fixiert, wie leicht einzusehen ist, wenn man die $\mathrm{zu}(42)$ gehörigen Graphen zeichnet:
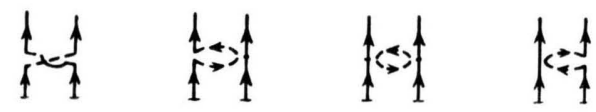

Abb. 10.

Die Übergangsamplitude wird in dieser Näherung

$$
\begin{aligned}
A= & -\frac{l^{4}}{4} \int \mathrm{d}^{4} x \int \mathrm{d}^{4} y \bar{\varphi}_{\mu \nu}^{(0) \prime}(x, y \mid) \\
& \cdot\left[S_{\mu \sigma}(x-y) S_{\sigma \varrho}(y-x) \varphi_{\varrho \nu}^{(0)^{\prime}}(x, y \mid)-S_{\varrho \sigma}(x-y)\right. \\
& \cdot S_{\sigma \varrho}(y-x) \varphi_{\mu \nu}^{(0)}(x, y \mid)+S_{\varrho \sigma}(x-y) S_{\nu \varrho}(y-x) \\
& \left.\cdot \varphi_{\mu \sigma}^{(0)}(x, y \mid)-S_{\mu \sigma}(x-y) S_{\nu \varrho}(y-x) \varphi_{\varrho \sigma}^{(0)}(x, y \mid)\right] .
\end{aligned}
$$

Man erkennt aus diesem Ausdruck unmittelbar, daß auch Kräfte langer Reichweite bei der Streuung wirksam sind. Denn die Funktion $S_{\mathrm{F}}(x-y)$ verhält sich bei großen raumartigen Abständen wie $(x-y)_{v} \gamma_{\nu} /(x-y)^{2}$, Produkte von zwei solchen $S_{\mathrm{F}}$-Funktionen nehmen also wie $1 /(x-y)^{2}$ ab, d.h. genau so wie die Schwingersche Funktion $\Delta_{\mathrm{F}}$. Die Streuamplitude verhält sich also bei kleinen Impulsänderungen qualitativ so, als ob die Streuung durch Lichtquanten vermittelt würde.

\section{b) Bose-Teilchen der Ruhmasse Null}

Die Ergebnisse des vorigen Abschnitts legen die Vermutung nahe, daß es in dem behandelten Modell einer Theorie der Elementarteilchen Bosonen der Ruhmasse Null geben muß - im Gegensatz zu einer in l. c. II geäußerten Vermutung. Wir wollen im folgenden zeigen, daß sich diese Teilchen tatsächlich aus den Grundgleichungen von II ergeben; und zwar läßt sich dies in der Näherung $N=2$ streng nachweisen und in den komplizierteren höheren Näherungen wenigstens plausibel machen.

Wir beginnen mit den Gleichungen für $N=2$ [vgl. II, Gl. (59)]:

$$
\gamma_{\mu}^{\alpha \beta} \frac{\partial \tau_{\beta \gamma}(x \mid y)}{\partial x_{\mu}}=-l^{2} \tau_{\alpha \delta \gamma \delta}(x x \mid y x)
$$

und $\quad \varphi_{\alpha \delta y \delta}(x x \mid y x)=0=\tau_{\alpha \delta \gamma \delta}(x x \mid y x)$

$$
+\frac{1}{2} S_{\alpha{ }^{\prime}}(x-y) \tau_{\delta \delta}(x \mid x)-\frac{1}{2} S_{\delta \gamma}(x-y) \tau_{\alpha \delta}(x \mid x) .
$$

Setzt man wieder

$$
\tau_{\alpha \beta}(x \mid y)=e^{i . J_{\mu} x_{\mu}} \tau_{\alpha \beta}(z)
$$

und geht zum Impulsraum über, so kann man die Gl. (44) in der üblichen Weise integrieren und erhält

$$
\begin{aligned}
\tau(z)= & \frac{1}{2(2 \pi)^{4}} \int \mathrm{d}^{4} p \frac{i l^{2} \gamma_{\mu}\left(J_{\mu}-p_{\mu}\right)}{\left(J_{\lambda}-p_{\lambda}\right)^{2}} \\
& \cdot(\tau(0)-\operatorname{Spur} \tau(0)) S(-p) e^{i p_{\mu} z_{\mu} .}
\end{aligned}
$$

Diese Formel gibt für $z=0$ ein System von 16 linearen Gleichungen mit $16 \mathrm{Un}$ bekannten $\tau_{\alpha \beta}(0)$; durch Nullsetzen der Determinante erhält man die Eigenwerte, d. h. zu einem Wertetripel $J_{1}, J_{2}$,

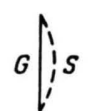

Abb. 11. $J_{3}$ den zugehörigen Wert $J_{4}$. Graphenmäßig wird (47) durch Abb. 11 dargestellt.

Für $S(p)$ wollen wir den Näherungswert [vgl. z.B. l. c. II, Gl. (31) und (33)]

$S(p)=2\left[\frac{p_{\mu} \gamma_{\mu} \varkappa^{4}}{\left(p_{\varrho}{ }^{2}\right)^{2}\left(p_{\sigma}{ }^{2}+\varkappa^{2}\right)}-\frac{i \varkappa^{3}}{p_{\varrho}{ }^{2}\left(p_{\sigma}{ }^{2}+\varkappa^{2}\right)}\right]$

mit $\varkappa=7,4 / l$ benützen.' Der exakte Wert von $S(p)$ wird von (48) etwas abweichen, wird sich aber in der Nähe von $p_{\varrho}{ }^{2}=0$ auch wie const $\cdot p_{\mu} \gamma_{\mu} /\left(p_{\varrho}{ }^{2}\right)^{2}$ verhalten, da dies aus dem Weglassen $\operatorname{der} \delta$-Funktionen auf dem Lichtkegel folgt.

Das Integral in (47) ist zunächst in der üblichen Weise auszurechnen. Man kann etwa zu $p_{\varrho}{ }^{2}$ und $\left(J_{\lambda}-p_{\lambda}\right)^{2}$ im Nenner ein Glied $-i \delta$ hinzufügen, in $p_{0}$ auf der reellen Achse integrieren und am Schluß zum limes $\delta \rightarrow 0$ übergehen. Auf diese Weise kann man sicher sein, daß man die Funktionen $G_{\mathrm{F}}$ und $S_{\mathrm{F}}$ genommen und die Randbedingungen und die Kausalitätsforderungen richtig berücksichtigt hat.

Dieses Verfahren ist jedoch aus zwei Gründen problematisch. Erstens zeigt eine Abzählung der Potenzen von $p$ in (47) im Zähler und Nenner, daß für $J_{\lambda}{ }^{2}=0$ das Integral für beliebige $\tau(0)$ im allgemeinen bei $p_{\varrho}=0$ logarithmisch divergiert. $Z_{w e i-}$ tens fallen zwei Pole des Integranden dann zusammen, wenn die räumliche Komponente von $p_{\lambda}$ zur räumlichen Komponente von $J_{\lambda}$ parallel und außer$\operatorname{dem} J_{\lambda}{ }^{2}=0$ ist. Anschaulich bedeutet das Zusammenfallen der Pole, daß der Zustand $J_{\lambda}{ }^{2}=0$ in der Weise realisiert werden kann, daß zwei virtuelle Fermionen der Ruhmasse Null parallel laufen und zusammen den Energie-Impulsvektor $J_{\lambda}$ geben. 
a) Die für $J_{\lambda}^{2}=0$ auftretenden Divergenzen

Eine Lösung der Gl. (47) im Falle $J_{\lambda}{ }^{2}=0 \mathrm{kann}$ es jedenfalls nur dann geben, wenn $\tau(0)$ so gewählt werden kann, daß die genannten Divergenzen verschwinden. Dazu ist zunächst einmal nötig, daß die Glieder mit $J_{\mu} \gamma_{\mu}$ im Zähler verschwinden. Es muß also

$$
J_{\mu} \gamma_{\mu}(\tau(0)-\operatorname{Spur} \tau(0))=0
$$

gefordert werden. Da man im Anfang auch nach $y$ statt nach $x$ hätte differenzieren können, muß auch

$$
(\tau(0)-\operatorname{Spur} \tau(0)) J_{\mu} \gamma_{\mu}=0
$$

gelten. Dies folgt zwar an dieser Stelle noch nicht zwangsläufig aus dem Rechnungsgang; es wird sich aber später unabhängig von der eben genannten Symmetrieüberlegung herausstellen, daß (50) eine notwendige Voraussetzung für die Konvergenz der Integrale (47) ist.

Die beiden Gln. (49) und (50) lassen sich in der Tat erfüllen. Setzt man in der üblichen Weise für die Diracschen Matrizen

$$
\gamma_{1,2,3}=-\varrho_{2} \sigma_{1,2,3} ; \quad \gamma_{4}=\varrho_{3} ; \quad \alpha_{1,2,3}=\varrho_{1} \sigma_{1,2,3}
$$

und wählt eine Darstellung, in der $\varrho_{1}$ die Form

hat, nimmt ferner

$$
\varrho_{1}=\left|\begin{array}{rrrr}
1 & 0 & 0 & 0 \\
0 & 1 & 0 & 0 \\
0 & 0 & -1 & 0 \\
0 & 0 & 0 & -1
\end{array}\right|
$$

an, so wird

$$
J_{1}=J_{2}=0 ; \quad J_{3}=J_{0}=J
$$

$$
\gamma_{\mu} J_{\mu}=i \gamma_{4} J\left(1+\alpha_{3}\right)=\left(1-\alpha_{3}\right) J \gamma_{4} i
$$

und jede Matrix $\tau(0)$, deren Elemente an allen außer den in (55) angekreuzten Stellen verschwinden, befriedigt die Gln. (49) und (50).

$$
\tau(0)=\left|\begin{array}{llll}
0 & 0 & 0 & 0 \\
\times & 0 & 0 & \times \\
\times & 0 & 0 & \times \\
0 & 0 & 0 & 0
\end{array}\right|
$$

Man erhält also vier linear unabhängige Lösungen von (49) und (50), die man auch in der Form

$$
\begin{aligned}
& \tau(0)=\left(1-\alpha_{3}\right)\left(\sigma_{1} \pm i \sigma_{2}\right) \text { und } \\
& \tau(0)=\left(1-\alpha_{3}\right)\left(\varrho_{2} \pm i \varrho_{3}\right)
\end{aligned}
$$

schreiben kann. Es wird sich später herausstellen, daß die ersten beiden Lösungen vektoriellen Bosonen entsprechen, d. h. als rechts- bzw. linkszirkulare Lichtwelle aufgefaßt werden können. Die beiden anderen Lösungen würden skalaren Bosonen entsprechen, aber für diese Werte von $\tau(0)$ bleiben, wie sich weiter unten zeigen wird, in dem Integral (47) noch Divergenzen stehen, es lassen sich aus ihnen also keine Lösungen von (47) herleiten. Bevor die Transformationseigenschaften der Ansätze
(56) untersucht werden, soll nun nachgerechnet werden, unter welchen Bedingungen sich aus ihnen Lösungen von (47) ergeben.

\section{$\beta)$ Das Verhalten der Lösungen}

Für die Bestimmung von $\tau(z)$ handelt es sich nach (47) und (48) im wesentlichen um die Berechnung der folgenden beiden Integrale unter der Voraussetzung $J_{\hat{\lambda}}^{2}=0$ :

$$
\begin{aligned}
A= & \lim _{\delta \rightarrow 0} \int \mathrm{d}^{4} p \\
& \cdot \frac{p_{\mu} \gamma_{\mu} \tau(0) p_{\sigma} \gamma_{\sigma}}{\left[\left(J_{\lambda}-p_{\lambda}\right)^{2}-i \delta\right]\left[p_{\varrho}{ }^{2}-i \delta\right]^{2}\left[p_{\sigma}^{2}+\varkappa^{2}-i \delta\right]} e^{i p_{\mu} z_{\mu}}
\end{aligned}
$$

und

$$
\begin{aligned}
B= & \lim _{\delta \rightarrow 0} \int \mathrm{d}^{4} p \\
\cdot & \frac{p_{\mu} \gamma_{\mu} \tau(0)}{\left[\left(J_{\lambda}-p_{\lambda}\right)^{2}-i \delta\right]\left[p_{\varrho}{ }^{2}-i \delta\right]\left[p_{\sigma^{2}}{ }^{2}+\varkappa^{2}-i \delta\right]} e^{i p_{\mu} z_{\mu}} .
\end{aligned}
$$

Die Rechnung soll nur für $A$ vorgeführt werden. Man erhält durch eine einfache Umformung:

$$
\begin{aligned}
A= & \lim _{\delta \rightarrow 0} \int_{0}^{\infty} \alpha \mathrm{d} \alpha \mathrm{d} \beta \mathrm{d} \gamma \int \mathrm{d}^{4} p p_{\mu} \gamma_{\mu} \tau(0) p_{\sigma} \gamma_{\sigma} \\
& \cdot \exp \left\{i p_{\mu} z_{\mu}-i \alpha\left(p_{\varrho}{ }^{2}-i \delta\right)-i \beta\left[\left(p_{\lambda}-J_{\lambda}\right)^{2}-i \delta\right]\right. \\
& \left.-i \gamma\left[p_{\sigma}{ }^{2}+\varkappa^{2}-i \delta\right]\right\}
\end{aligned}
$$

und unter Benützung von (49) und (50) (hier ist auch (50) eine notwendige Bedingung der Konvergenz!)

$$
\begin{aligned}
A= & \lim _{\delta \rightarrow 0}\left(-i \pi^{2}\right) \int_{0}^{\infty} \frac{\alpha \mathrm{d} \alpha \mathrm{d} \beta \mathrm{d} \gamma}{(\alpha+\beta+\gamma)^{4}} \\
& \cdot\left[\frac{\alpha+\beta+\gamma}{2} \gamma_{\mu} \tau(0) \gamma_{\mu}+\frac{1}{4} z_{\mu} \gamma_{\mu} \tau(0) z_{\sigma} \gamma_{\sigma}\right] \\
& \cdot \exp \left\{-\delta(\alpha+\beta+\gamma)-i \gamma \varkappa^{2}+i \frac{z_{\varrho}{ }^{2}+4 \beta J_{\varrho} z_{\varrho}}{4(\alpha+\beta+\gamma)}\right\} .
\end{aligned}
$$

Man erkennt, daß das Integral für $\delta \rightarrow 0$ immer noch logarithmisch divergiert, sofern $\gamma_{\mu} \tau(0) \gamma_{\mu}$ nicht verschwindet. Es muß also noch

gefordert werden.

$$
\gamma_{\mu} \tau(0) \gamma_{\mu}=0
$$

Tatsächlich verschwindet dieser Ausdruck für die ersten Lösungen (56), d. h. für

$$
\tau(0)=\left(1-\alpha_{3}\right)\left(\sigma_{1} \pm i \sigma_{2}\right),
$$

aber nicht für die beiden anderen. Wir können also jedenfalls nur für den Ansatz (62) Lösungen der Ausgangsgleichungen zum Eigenwert $J_{\lambda}^{2}=0$ erhalten. Unter der Voraussetzung (62) kann man nun das Verhalten des Integrals $A$ bei kleinen und großen Werten von $z_{\mu}$ leicht diskutieren und erhält 
$A \sim$ const $\cdot z_{\mu} \gamma_{\mu} \tau(0) z_{\sigma} \gamma_{\sigma} \cdot \lg \left(z_{\varrho}^{2}\right)$

für kleine $z_{\varrho}\left(z_{\varrho} J_{\varrho} \ll 1 ; \quad z_{\varrho}^{2} x^{2} \ll 1\right)$, $A \sim$ const $\cdot \frac{z_{\mu} \gamma_{\mu} \tau(0) z_{\sigma} \gamma_{\sigma}}{\left(z_{\varrho}\right)^{2}} \frac{e^{i J_{\varrho} z_{\varrho}}-1-i\left(J_{\varrho} z_{\varrho}\right)}{\left(J_{\varrho} z_{\varrho}\right)^{2}}$

für große $z_{\mu}{ }^{2}\left(z_{\mu}{ }^{2} \varkappa^{2} \gg 1\right)$.

Eine entsprechende Rechnung für $B$ ergibt

$B \sim$ const $\cdot z_{\mu} \gamma_{\mu} \tau(0) \lg \left(z_{\varrho}{ }^{2}\right) \quad$ für kleine $z_{\varrho}$,

$B \sim$ const $\cdot \frac{z_{\mu} \gamma_{\mu} \tau(0)}{\left(z_{\varrho}{ }^{2}\right)^{2}} \frac{e^{i J_{\varrho} z_{\varrho}}-1}{J_{\varrho} z_{\varrho}}$ für große $z_{\varrho}{ }^{2}$.

Diese Ergebnisse zeigen, daß man jetzt zunächst auf der linken Seite von (47) $\tau(z)=0$ für $z=0$ erhält, also ein Resultat, das zu (62) in Widerspruch steht.

Dabei ist aber eine Freiheit noch nicht ausgenützt worden, die mit dem gelegentlichen Zusammenfallen der beiden Pole des Integranden in (47) zusammenhängt. Man kann diese Freiheit in doppelter Weise ausdrücken.

Man kann erstens feststellen, daß man in (57) und (58) vor dem Grenzübergang $\delta \rightarrow 0$ noch allgemeinere Ansätze für $\tau(0)$ als (56) machen kann, denn vor dem Grenzübergang konvergieren alle Integrale. Man muß nur dafür sorgen, daß $\tau(0)$ im limes $\delta \rightarrow 0$ in (62) übergeht. Tut man dies, so erhält man von solchen Gliedern Beiträge zu $\tau(z)$ auf der linken Seite von (47), die für sich allein einfach die Gleichung

$$
\gamma_{\mu} \frac{\partial \tau(x \mid y)}{\partial x_{\mu}}=0
$$

befriedigen. Diese Terme kann man also dazu ausnützen, die Gl. (47) zu erfüllen.

Die Durchführung der Rechnung gibt zusätzlich zu $A$ ein (für $\delta \rightarrow 0$ divergentes) Integral, das nach der Umformung die Gestalt

$$
\begin{aligned}
C= & \text { const } \cdot \gamma_{\sigma} J_{\sigma} \cdot \tau(0) \int \frac{\alpha \mathrm{d} \alpha \mathrm{d} \beta \mathrm{d} \gamma}{(\alpha+\beta+\gamma)^{3}}\left(z_{\varrho} \gamma_{\varrho}+2 \beta J_{\varrho} \gamma_{\varrho}\right) \\
& \cdot \exp \left\{-\delta(\alpha+\beta+\gamma)-i \chi^{2} \gamma+i \frac{z_{\varrho}{ }^{2}+4 \beta z_{\varrho} J_{\varrho}}{(\alpha+\beta+\gamma)}\right\}
\end{aligned}
$$

erhält. Der für $\delta \rightarrow 0$ größte Anteil des Integrals wird

$$
\begin{aligned}
C \approx & \text { const } \cdot \gamma_{\sigma} J_{\sigma} \tau(0) J_{\varrho} \gamma_{\varrho} \int \frac{\alpha \mathrm{d} \alpha \beta \mathrm{d} \beta}{(\alpha+\beta)^{3}} \\
& \cdot \exp \left\{-\delta(\alpha+\beta)+i \frac{\beta}{\alpha+\beta} J_{\varrho} z_{\varrho}\right\} \\
\approx & \frac{\gamma_{\sigma} J_{\sigma} \tau(0) J_{\varrho} \gamma_{\varrho}}{\delta} \cdot \mathrm{const} \\
& \cdot \exp \left\{\frac{1}{2} i J_{\varrho} z_{\varrho}\right\} \frac{\sin \frac{1}{2} J_{\varrho} z_{\varrho}-\frac{1}{2} J_{\varrho} z_{\varrho} \cos \frac{1}{2} J_{\varrho} z_{\varrho}}{\left(J_{\varrho} z_{\varrho}\right)^{3}} .
\end{aligned}
$$

Wenn man zu $\tau(0)$ also ein Glied fügt, das vor dem Grenzübergang $\delta \rightarrow 0$ von der Größenordnung $\delta$ ist und das nicht die Form (56) hat, so erhält man einen Zusatz zu $\tau(z)$ in (47), der nur von $J_{\varrho} z_{\varrho}$ abhängt, die Bedingung $\gamma_{\sigma} \frac{\partial \tau(z)}{\partial z_{\sigma}}=0$ befriedigt und dazu ausgenützt werden kann, Gl. (47) zu erfüllen.

Als vollständige Lösung der Gln. (44) und (45) ergibt sich daher schließlich:

$$
\begin{aligned}
& \tau(z)=\left(1-\alpha_{3}\right)\left(\sigma_{1} \pm i \sigma_{2}\right) \\
& \cdot \exp \left\{\frac{1}{2} i J_{\varrho} z_{\varrho}\right\} 24 \frac{\sin \frac{1}{2} J_{\varrho} z_{\varrho}-\frac{1}{2} J_{\varrho} z_{\varrho} \cos \frac{1}{2} J_{\varrho} z_{\varrho}}{\left(J_{\varrho} z_{\varrho}\right)^{3}} \\
& +\frac{1}{2} \int \mathrm{d}^{4} p \frac{(-i l)^{2} \gamma_{\mu} p_{\mu}}{\left(J_{\lambda}-p_{\lambda}\right)^{2}}\left(1-\alpha_{3}\right)\left(\sigma_{1} \pm i \sigma_{2}\right) \\
& \cdot S(-p) e^{i p_{\mu} z_{\mu}} \cdot \frac{1}{(2 \pi)^{4}} .
\end{aligned}
$$

Die zweite Art, die erwähnte Freiheit im Integral (47) auszudrücken, besteht in der Feststellung, daß überall dort, wo die beiden Pole des Integranden zusammenfallen, der Integrationsweg um den Pol durch die Randbedingungen nicht mehr festgelegt ist, da die beiden Pole ursprünglich von verschiedenen Seiten umgangen werden sollten. Man kann also Beiträge, die dieser Lage der Pole entsprechen, zu $\tau(z)$ hinzufügen; diese Beiträge genügen dann immer der Bedingung $\gamma_{\mu} \partial \tau(x \mid y) / \partial x_{\mu}=0$. Mit dieser Schlußweise würde man noch etwas allgemeinere Lösungen von (44) und (45) als (47) zulassen können, nämlich

$$
\begin{aligned}
\tau(z)= & \left(1-\alpha_{3}\right)\left(\sigma_{1} \pm i \sigma_{2}\right) f\left(J_{\mu} z_{\mu}\right) \\
& +\frac{1}{2} \int \mathrm{d}^{4} p \frac{\left(-i l^{2}\right) \gamma_{\mu} p_{\mu}}{\left(J_{\lambda}-p_{\lambda}\right)^{2}}\left(1-\alpha_{3}\right)\left(\sigma_{1} \pm i \sigma_{2}\right) f(0) \\
& S(-p) e^{i p_{\mu} z_{\mu}} \cdot \frac{1}{(2 \pi)^{4}},
\end{aligned}
$$

wobei $f\left(J_{\mu} z_{\mu}\right)$ irgendeine beliebige Funktion von $J_{\mu} z_{\mu}$ ist. Daß die Funktion $f\left(J_{\mu} z_{\mu}\right)$ hier nicht weiter festgelegt wird, ist im Grunde verständlich, da in der Näherung $N=2$ nur $\tau(x \mid x)$ durch eine Integralgleichung bestimmt wird, wie schon das Graphenbild Abb. 11 zeigt. Erst in höheren Näherungen, in denen man auch Graphen berücksichtigt, an deren Endpunkten $x$ und $y$ in $\tau(x \mid y)$ verschieden sind, wird die Funktion $f\left(J_{\mu} z_{\mu}\right)$ festgelegt werden können.

Anschaulich bedeutet das erste Glied in (67) bzw. (68), daß zwei virtuelle Fermionen der Ruhmasse Null genau parallel laufen und zusammen den Vektor $J_{\mu}$ ergeben. Man könnte daher zunächst glauben, daß es sich bei den Zuständen (68) gar 
nicht um echte gebundene Zustände handelte, sondern daß hier eben zwei Fermionen sozusagen zufällig nebeneinander herlaufen. Eine solche Interpretation gibt aber die Verhältnisse nicht richtig wieder, da ja die Wechselwirkung berücksichtigt ist, es also dann eine Streuwelle geben müßte. Die Ausdrücke (63) und (64) können aber nicht als Streuwelle gedeutet werden. Außerdem müßte es dann vier und nicht nur zwei Zustände geben, den vier möglichen Spinrichtungen entsprechend. Schließlich kommen in der hier besprochenen Modelltheorie keine wirklichen Fermi-Teilchen der Ruhmasse Null vor, was sich allerdings erst in höheren Näherungen, von $N=3$ ab, zeigt. Es handelt sich also bei den Zuständen (68) um echte diskrete stationäre Zustände, da es kein Kontinuum solcher Zustände gibt, nur weisen die Eigenfunktionen etwas ungewöhnliche Eigenschaften auf, die in einigen Punkten an die Eigenfunktionen von Streuzuständen erinnern.

\section{$(\gamma)$ Die Transformationseigenschaften der Lösungen}

Um die Transformationseigenschaften der Lösungen (62) zu studieren, wird es genügen, eine infinitesimale Lorentz-Transformation in der $x_{1^{-}}$ Richtung vorzunehmen. Wir betrachten also das in der $x_{3}$-Richtung sich bewegende Boson von einem Koordinatensystem $K^{\prime}$ aus, das sich mit der Geschwindigkeit $\beta(\beta \ll 1)$ in der positiven $x_{1}$-Richtung bewegt. In diesem Koordinatensystem wird, unter Vernachlässigung aller höherer Glieder in $\beta$,

$$
J_{1}{ }^{\prime}=-\beta J ; J_{2}{ }^{\prime}=0 ; J_{3}{ }^{\prime}=J ; J_{0}{ }^{\prime}=J .
$$

Eine infinitesimale Drehung in der $x_{1}, x_{4}$-Ebene um den Winkel $i \beta$ entspricht im Spinorraum einer kanonischen Transformation nach der Formel:

$$
\begin{aligned}
T^{\prime} & =\left(1-\frac{i \beta}{2} \gamma_{1} \gamma_{4}\right) T\left(1+\frac{i \beta}{2} \gamma_{1} \gamma_{4}\right) \\
& =\left(1+\frac{\beta}{2} \alpha_{1}\right) T\left(1-\frac{\beta}{2} \alpha_{1}\right) .
\end{aligned}
$$

Setzt man für $T$ die Matrix $\tau(0)$ aus (62) ein, so folgt

$$
\begin{aligned}
\tau^{\prime}(0) & =\left(1+\frac{\beta}{2} \alpha_{1}\right)\left(1-\alpha_{3}\right)\left(\sigma_{1} \pm i \sigma_{2}\right)\left(1-\frac{\beta}{2} \alpha_{1}\right) \\
& =\left(1-\alpha_{3}+\beta \alpha_{1}\right)\left(\sigma_{1}+\beta \sigma_{3} \pm i \sigma_{2}\right) .
\end{aligned}
$$

Nun bedeutet $\sigma_{3}-\beta \sigma_{1}$ gerade die Komponente des Spins in der Fortpflanzungsrichtung des Bosons in $K^{\prime}, \sigma_{1}+\beta \sigma_{3}$ die Komponente in der dazu (und zur $x_{2}$-Richtung) senkrechten Richtung. $\tau^{\prime}(0)$ hat also in bezug auf die neue Fortpflanzungsrichtung des Bosons genau die gleiche Form wie $\tau(0)$ in bezug auf die alte, d. h. die Lösung (62) wird bei einer Lorentz-Transformation in sich transformiert. Das ist genau das Verhalten einer zirkular polarisierten Lichtwelle; die rechtszirkulare Welle geht z. B. bei einer Lorentz-Transformation wieder in eine rechtszirkulare Welle über.

Daß die Lösungen (62) den zirkularpolarisierten Lichtwellen entsprechen, erkennt man auch durch eine Berechnung des zugehörigen Drehimpulses. Z.B. bedeutet

$$
\tau(0)=\left(1-\alpha_{3}\right)\left(\sigma_{1}+i \sigma_{2}\right)
$$

bei der gewählten Darstellung die Matrix

$$
\tau(0)=\left|\begin{array}{llll}
0 & 0 & 0 & 0 \\
0 & 0 & 0 & 0 \\
0 & 0 & 0 & 4 \\
0 & 0 & 0 & 0
\end{array}\right| .
$$

Da $\tau_{\alpha \beta}(0)$ dem Element $\left\langle\Omega\left|\psi_{\alpha}(x) \psi_{\beta}^{+}(x)\right| \Phi\right\rangle$ entspricht und $\psi$ bzw. $\psi^{+}$die Operatoren für die Vernichtung bzw. Erzeugung eines virtuellen Spinorteilchens darstellen, so bewirkt $\psi_{\alpha}(x) \psi_{\beta}^{+}(x)$ für das Matrixelement (73) die Vernichtung eines Teilchens vom Spin $\sigma_{3}=1$ und die Erzeugung eines Teilchens vom Spin $\sigma_{3}=-1$ mit negativer Energie; das letztere bedeutet Vernichtung eines Antiteilchens positiver Energie vom Spin $\sigma_{3}=+1$. Der Zustand $\Phi$ enthält also je ein virtuelles Teilchen und Antiteilchen vom Spin $\sigma_{3}=1$; der zugehörige Drehimpuls um die $x_{3}$-Achse ist daher $2 \cdot \hbar / 2=\hbar$. Entsprechend gehört zu

$$
\tau(0)=\left(1-\alpha_{3}\right)\left(\sigma_{1}-i \sigma_{2}\right)
$$

der Drehimpuls $-\hbar$ um die $x_{3}$-Achse.

Wenn man zwei Spinorteilchen endlicher Masse durch Kräfte zu einem Bose-Teilchen endlicher Masse verbindet, so ergeben sich vier Zustände. Bei einer Lorentz-Transformation werden die drei Zustände, die zum Gesamtdrehimpuls $\hbar$ gehören, ineinander transformiert, der letzte Zustand mit dem Drehimpuls 0 geht in sich über. Daß im Gegensatz dazu hier jeder Zustand in sich transformiert wird, liegt an der Besonderheit, daß bei einem Teilchen der Ruhmasse Null eine infinitesimale Lorentz-Transformation senkrecht zur Bewegungsrichtung einer von der Energie des Bosons unabhängigen Drehung im dreidimensionalen Raum äquivalent ist. Eben dieser Umstand ermöglicht es auch, daß hier statt der vier Zustände nur zwei auftreten. 


\section{$\delta)$ Die höheren Näherungen}

Die Existenz der Lösungen zum Eigenwert $J_{\lambda^{2}}=0$ beruht in der Näherung $N=2$ auf der Besonderheit, $\mathrm{da} B$ in den Integralgleichungen gewisse Impulsintegrale zunächst divergieren, daß also der Ausgangsfunktion $\tau(x, y)$ Bedingungen auferlegt werden müssen, um die Divergenzen zu beseitigen; diese Bedingungen lassen sich erfüllen. Nachdem die Bedingungen erfüllt sind, kann die Integralgleichung ohne Schwierigkeit gelöst werden, indem man sozusagen durch infinitesimale Abweichungen der Ausgangsfunktionen von den durch die Konvergenzbedingung gegebenen Werten die linke Seite gleich der rechten macht. Im Ortsraum bedeutet dies, daß die Lösungen $\tau(z)$ sich im Unendlichen anders verhalten, als dies sonst für die Eigenfunktionen diskreter Zustände üblich ist; dieses andersartige Verhalten kann, wenn man es überhaupt anschaulich interpretieren will, als Folge einer in $\operatorname{der} S_{\mathrm{F}^{-}}$ Funktion ausgedrückten ,,Kraft“ zwischen den virtuellen Spinorteilchen aufgefaßt werden, die noch langsamer als eine Coulomb-Kraft abfällt.

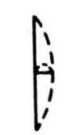

Abb. 12 .

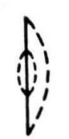

Abb. 13 .

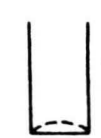

Abb. 14 .
Man kann einsehen, daß die hier geschilderten Besonderheiten in den höheren Näherungen genau so auftreten, wie in der bisher behandelten niedrigsten. Für Graphen von der durch die Abb. 12 und 13 dargestellten Art folgt dies sofort aus ihrer zu Abb. 9 ähnlichen Struktur. Aber auch Graphen ganz anderer Art sind mit dem in (63), (64) und (68) ausgedrückten asymptotischen Verhalten verträglich. Schreibt man z. B. das durch Abb. 14 angedeutete System als Differentialgleichung auf, so entsteht, wenn man

$$
\tau(x, y)=\vartheta(z) \exp \left\{\frac{1}{2}-i J_{\mu}\left(x_{\mu}+y_{\mu}\right)\right\}
$$

setzt, für $\vartheta(z)$ die Gleichung

$$
\begin{aligned}
& 4 \gamma_{\mu}\left(\frac{i J_{\mu}}{2}-\frac{\vec{\partial}}{\partial z_{\mu}}\right) \vartheta(z)\left(\frac{i J_{\nu}}{2}+\frac{\overleftarrow{\partial}}{\partial z_{\nu}}\right) \gamma_{\nu} \\
&=-l^{4} S(-z) \operatorname{Spur}[S(z) \vartheta(z)] \\
&+l^{4} S(-z) S(z) \vartheta(z) \\
&-l^{4} S(-z) \operatorname{Spur}[S(-z) \vartheta(-z)] \\
&+l^{4} S(-z) \vartheta(-z) S(-z) \\
&-l^{4} \vartheta(z) \operatorname{Spur}[S(z) S(-z)] \\
&+l^{4} \vartheta(z) S(z) S(-z) .
\end{aligned}
$$

Setzt man für $\vartheta(z)$ wieder als größtes Glied an

$$
\underset{z \rightarrow \infty}{\vartheta(z)}=\left(1-\alpha_{3}\right)\left(\sigma_{1} \pm i \sigma_{2}\right) f\left(J_{\mu} z_{\mu}\right)+\ldots,
$$

so erkennt man, daß die nächsten Glieder etwa die Form

$$
\begin{aligned}
& \text { const } \cdot \frac{\left(\sigma_{1} \pm i \sigma_{2}\right)}{z_{\varrho}{ }^{2}} f\left(J_{\mu} z_{\mu}\right) \quad \text { oder } \\
& \text { const } \cdot \frac{\gamma_{\nu} z_{\nu}\left(\sigma_{1} \pm i \sigma_{2}\right) \gamma_{\lambda} z_{\lambda}}{\left(z_{\varrho}{ }^{2}\right)^{2}} f\left(J_{\mu} z_{\mu}\right)
\end{aligned}
$$

haben müssen und daß diese Glieder auf der linken Seite von (74) gerade die größten Glieder der rechten Seite kompensieren. Man hat also den Eindruck, daß die Lösungen (68) auch in den höheren Näherungen qualitativ bestehen bleiben; ein wirklicher Beweis dafür könnte aber wohl nur durch eine viel genauere Untersuchung geliefert werden und soll hier nicht versucht werden.

c) Höhere Näherungen für die Kraft

zwischen zwei Spinorteilchen und Berechnung der,,Feinstrukturkonstante“

Die Überlegungen des vorausgehenden Abschnitts legen es nahe, bei der Berechnung der Kräfte langer Reichweite neben den in 2 a) behandelten Graphen der Form <__- auch noch die zopfartigen Graphen der allgemeinen Form

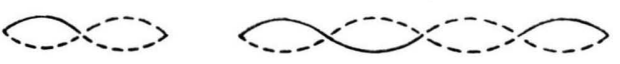

usw.

mit zu berücksichtigen, die wegen des in 2 b) besprochenen Verhaltens bei $J_{u}{ }^{2} \rightarrow 0$ keineswegs nur kleine Beiträge zu dem Integralkern $K_{\mathrm{W}}$ liefern, sondern diesen entscheidend verändern. Es wird an dieser Stelle sogar notwendig sein, alle Beiträge dieser Art aufzusummieren, um eine brauchbare erste Näherung für den Integralkern zu erhalten, den wir graphenmäßig durch $K$ Abb.15. andeuten wollen. Wir denken also an Graphen einer sehr hohen Näherung, bei denen zunächst sehr lange $G$-Linienzüge von den beiden Selbstenergieteilen zu der Stelle der Wechselwirkung ¿..._> reichen. Wenn diese $G$-Linienzüge fixiert sind, ist über alle möglichen $S$-Linienbilder zu summieren. Das einzelne $S$-Linienbild enthält dann immer zwei (in Abb. 16 durch Ovale angedeutete)

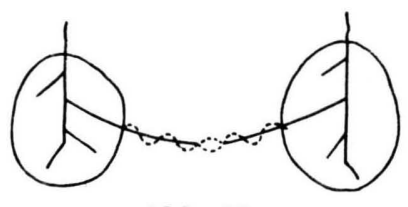

Abb. 16.

Selbstenergieteile und den dazwischenliegenden Zopf. Von den Selbstenergieteilen kann angenommen werden, daß sie einen Operator $O^{\text {s }}$ beisteuern, der schließlich, wenn man zu hinreichend hohen Näherungen übergeht, von der Höhe der Näherung praktisch unabhängig wird. Dann bleibt eine Summe über Zöpfe verschiedener Länge auszuführen, wobei irgendwo innerhalb des Zopfes die 
Stelle mit den zwei $S$-Linien vorkommt. Statt $K$ kann man also auch den Graphen

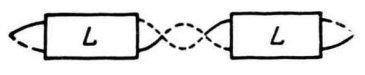

Abb. 17.

zeichnen, wobei nur noch $L$ zu berechnen ist.

Formelmäßig bedeuten die eben durchgeführten Überlegungen für den in 2 a) eingeführten Operator $N$

$$
\begin{aligned}
N_{\mu \nu \mid \varrho \sigma}\left(x y \mid x^{\prime} y^{\prime}\right) & =\delta\left(x-x^{\prime}\right) \delta\left(y-y^{\prime}\right) \\
& O_{\mu \varrho \mid \eta \xi}^{\mathrm{s}} K_{\eta \xi \mid<\lambda}\left(x^{\prime}, x^{\prime}\right) O_{* \lambda \mid \nu \sigma}^{\mathrm{s}}
\end{aligned}
$$

und für die Übergangsamplitude $A$

$$
\begin{aligned}
A= & -l^{4} \int \mathrm{d}^{4} x \mathrm{~d}^{4} y \bar{\varphi}_{\mu \nu}^{(0)}(x, y \mid) \\
& O_{\mu \varrho \mid \eta \xi}^{\mathrm{s}} K_{\eta \xi \mid \kappa \lambda}\left(x^{\prime}, y\right) O_{k \lambda \mid \nu \sigma}^{\mathrm{s}} \varphi_{\varrho \sigma}^{(0)}(x, y \mid) .
\end{aligned}
$$

In der untersten Näherung hatten wir in 2 a) für $K_{\mu \varrho \mid v \sigma}$ den Wert

$K_{\mu \varrho \mid \nu \sigma}(x, y)=\frac{1}{4}-S_{\alpha \beta}(x-y) S_{\gamma \delta}(y-x) M_{\alpha \varrho \mid \mu \delta} M_{\sigma \gamma \mid \nu \beta}$

erhalten, wobei

$$
M_{\alpha \mu \mid \gamma \delta}=\delta_{\alpha \gamma} \delta_{\beta \delta}-\delta_{\alpha \delta} \delta_{\beta \gamma}
$$

gesetzt ist. Für $O^{\mathrm{s}}$ würde in der untersten Näherung $O_{\mu \varrho \mid \eta \xi}^{\mathrm{s}}=\delta_{\mu \eta} \delta_{\varrho \xi}$ gelteiı. In der gesuchten höheren Näherung hat nunmehr $K$ die Form

$$
\begin{aligned}
K_{\mu \varrho \mid v \sigma}(x, y)= & \frac{1}{4} \int \mathrm{d}^{4} w \mathrm{~d}^{4} z \\
& \cdot\left[\delta_{\varrho \alpha} \delta_{\mu \beta} \delta(x-z)+l^{2} L_{\mu \beta \alpha} \alpha_{\varrho}(x, z)\right] \\
& \cdot S_{\varepsilon \varphi}(z-w) S_{\psi \eta}(w-z) M_{\varepsilon \alpha \mid \beta \eta} M_{\gamma \psi \mid \delta \varphi} \\
& \cdot\left[\delta_{\sigma \gamma} \delta_{\nu \delta} \delta(w-y)+l^{2} L_{\nu \delta \gamma \sigma}(w, y)\right] .
\end{aligned}
$$

Die Berechnung höherer Näherungen für $O^{\mathrm{s}}$ soll in dieser Arbeit noch nicht versucht werden.

Wir nehmen jetzt an, daß in dem Ausgangsgraphen (Abb. 16) die $G$-Linien einfach mit einheitlicher Pfeilrichtung durchverbunden sind. Das bedeutet eine sehr spezielle hohe Näherung; man hätte statt dessen auch über verschiedene derartige Näherungen mitteln können, doch hätte das die Rechnungen noch komplizierter gemacht. In dieser speziellen hohen Näherung kann man dann die Summation über die verschiedenen Zopflängen durchführen, indem man für $L_{\mu f \alpha \varrho}$ die Integralgleichung

$$
\begin{aligned}
4 L_{\mu \beta \alpha \varrho}(x, z) & =i G_{\mu \beta}(x-z) S_{\alpha \varrho}(z-x) \\
& +i l^{2} \int G_{\mu \tau}(x-w) S_{\lambda \varrho}(w-x) L_{\tau \beta \alpha \lambda}(w, z) \mathrm{d}^{4} w
\end{aligned}
$$

fordert.

Die weitere Rechnung erfolgt im Impulsraum:

$$
\begin{aligned}
K_{\mu \nu \mid \varrho \sigma}(x, y) & =\frac{1}{(2 \pi)^{8}} \int e^{i(p x+q y)} K_{\mu \nu \mid \varrho \sigma}(p, q) \mathrm{d}^{4} p \mathrm{~d}^{4} q \text { und } \\
S_{\alpha \beta}(x) & =\frac{1}{(2 \pi)^{4}} \int e^{i p x} S_{\alpha \beta}(p) \mathrm{d}^{4} p .
\end{aligned}
$$

Aus (81) wird dann

$$
\begin{aligned}
K_{\mu \varrho \mid \nu \sigma}(p, q \mid)= & \frac{1}{4} \int \mathrm{d}^{4} r \mathrm{~d}^{4} s \\
& \cdot\left[\delta_{\varrho \alpha} \delta_{\mu \beta} \delta(r+p)+\frac{l^{2}}{(2 \pi)^{4}} L_{\mu \beta \alpha \varrho}(p, r)\right] \\
& \cdot S_{\varepsilon \varphi}(s-p) S_{\psi \eta}(s) M_{\varepsilon \alpha \mid \beta \eta} M_{\gamma \psi \mid \delta \varphi} \\
& \cdot\left[\delta_{\varrho \gamma} \delta_{\nu \delta} \delta(q+r)+\frac{l^{2}}{(2 \pi)^{4}} L_{\nu \delta \gamma \sigma}(-r, q)\right] .
\end{aligned}
$$

Für die Übergangsamplitude folgt mit $O^{\mathrm{s}}{ }_{\mu \varrho} \mid \eta \xi=\delta_{\mu \eta} \delta_{\varrho \xi}$ : $A=-l^{4} \bar{u}_{\mu \nu}\left(p^{\prime}, q^{\prime} \mid\right) K_{\mu \varrho \mid \nu \sigma}\left(p^{\prime}-p, q^{\prime}-q\right) u_{\varrho \sigma}(p, q \mid)$. (85)

Die Integralgl. (82) schließlich erhält die Form

$4 L_{\mu \beta \alpha \varrho}(p, r)=H_{\mu \beta \alpha \varrho}(p) \delta(p+r)$

$$
+\frac{l^{2}}{(2 \pi)^{4}} H_{\mu \tau \lambda \varrho}(p) \cdot L_{\tau \beta \alpha \lambda}(p, r)
$$

mit

$$
H_{\mu \beta \alpha \varrho}(p)=i \int G_{\mu \beta}(p+r) S_{\alpha \varrho}(r) \mathrm{d}^{4} r ;
$$

dieser Operator ist praktisch identisch mit dem auf der rechten Seite von (47) für $z=0$ auftretenden Operator.

Das Integral wird wieder mit den Feynmanschen Methoden berechnet, und man findet

$H_{\mu \beta \alpha \varrho}(p)=4 \pi^{2} \varkappa^{3}\left[i(p \gamma)_{\mu \beta} \delta_{\alpha \varrho} N_{1}\right.$

$$
\left.-\frac{\varkappa}{2} \gamma_{\mu \beta}^{\sigma} \gamma_{\alpha \varrho}^{\sigma} N_{3}+\varkappa(p \gamma)_{\mu \beta}(p \gamma)_{\alpha \varrho} N_{2}\right] \text {, }
$$

wobei

$$
\begin{gathered}
N_{1}=\int_{0}^{1} \mathrm{~d} x \mathrm{~d} y \frac{x}{p^{2}(1-x)+\varkappa^{2} y} \\
=\frac{\left(p^{2}+\varkappa^{2}\right)^{2}}{2 \varkappa^{2} p^{4}} \lg \frac{p^{2}+\varkappa^{2}}{\varkappa^{2}}-\frac{1}{2 \varkappa^{2}} \lg \frac{p^{2}}{\varkappa^{2}}-\frac{1}{2 p^{2}}, \\
N_{2}=\int_{0}^{1} \mathrm{~d} x \mathrm{~d} y \frac{x(1-x)(1-y)}{\left[p^{2}(1-x)+\varkappa^{2} y\right]^{2}} \\
=\frac{1}{6 p^{2} \varkappa^{2}}-\frac{1}{3 p^{4}}+\frac{1}{6 \varkappa^{4}} \lg \frac{p^{2}}{\varkappa^{2}}-\frac{\left(p^{2}+\varkappa^{2}\right)^{2}}{p^{4} \varkappa^{4}} \\
N_{3}=\int_{0}^{1} \mathrm{~d} x \mathrm{~d} y \frac{x\left(1-\frac{\varkappa^{2}}{3 p^{2}}\right) \lg \frac{p^{2}+\varkappa^{2}}{\varkappa^{2}},}{(1-x)+\varkappa^{2} y} \\
=-\frac{1}{6 p^{2}}-\frac{1}{6 \varkappa^{2}}-\frac{1}{\varkappa^{4}}\left(\frac{p^{2}}{6}+\frac{\varkappa^{2}}{2}\right) \lg \frac{p^{2}}{\varkappa^{2}} \\
+\frac{\left(p^{2}+\varkappa^{2}\right)^{3}}{6 p^{4} \varkappa^{4}} \lg \frac{p^{2}+\varkappa^{2}}{\varkappa^{2}} .
\end{gathered}
$$

Das Verhalten dieser Funktionen bei kleinen $p$ ist durch

$$
\begin{aligned}
& N_{1} \approx-\frac{1}{2 \varkappa^{2}} \lg \frac{p^{2}}{\varkappa^{2}}+\text { const, } \\
& N_{2} \approx+\frac{1}{2 p^{2} \varkappa^{2}}+\frac{1}{6 \varkappa^{4}} \lg \frac{p^{2}}{\varkappa^{2}}+\text { const }, \\
& N_{3} \approx-\frac{1}{2 \varkappa^{2}} \lg \frac{p^{2}}{\varkappa^{2}}+\text { const }
\end{aligned}
$$

gegeben. 
Nun vereinfachen wir die Gleichung für $L$ durch den Ansatz

$$
\begin{aligned}
L_{\mu \beta \alpha \varrho}(p, r)= & -\frac{(2 \pi)^{4}}{l^{2}} \delta_{\mu \beta} \delta_{\alpha \varrho} \delta(p+r) \\
& +\delta(p+r) \frac{(2 \pi)^{4}}{l^{2}} Q_{\mu \beta \alpha \varrho}(p) .
\end{aligned}
$$

Dann folgt aus (86):

$$
Q_{\mu \beta \alpha \varrho}=\delta_{\mu \beta} \delta_{\alpha \varrho}+\frac{l^{2}}{4(2 \pi)^{4}} H_{\mu \tau \lambda \varrho} Q_{\tau \beta \alpha \lambda} .
$$

Bevor wir $Q_{\mu \propto \alpha \varrho}$ aus (92) ausrechnen, schreiben wir noch den Operator $K_{\mu \varrho \mid v \sigma}(p, q)$ auf; dabei berücksichtigen wir in $\boldsymbol{S}_{\alpha \beta}(p)$ jeweils nur die Glieder mit der niedrigsten Potenz von $p$, da nur diese zu den Kräften langer Reichweite Anlaß geben. Aus (84) und (91) folgt in dieser Näherung

$$
\begin{aligned}
& K_{\mu \varrho \mid \nu \sigma}=- \frac{i \pi^{2} \varkappa^{4}}{p^{2}} Q_{\nu \delta \gamma \sigma}(q) \\
& \cdot\left[\frac{1}{2}\left(\gamma_{\lambda}\right)_{\beta \gamma}\left(\gamma_{\lambda}\right)_{\delta \alpha}-\frac{(\gamma p)_{\beta \gamma}(\gamma p)_{\delta \alpha}}{p^{2}}+2 \delta_{\alpha \beta} \delta_{\gamma \delta}\right] \\
& \cdot Q_{\mu \beta \alpha \varrho}(p) \delta(p+q) .
\end{aligned}
$$

Bei der Berechnung von $Q$ aus dem linearen Gleichungssystem (92) ist nun wie in $(2 \mathrm{~b}, \alpha$ und $\beta) \mathrm{zu}$ beachten, daß gewisse Teile des Operators $H$ für $p^{2} \rightarrow 0$ unendlich groß werden. Man kann also $Q$ in zwei Teile spalten:

$$
Q=V+R
$$

der erste, $V$, soll so geartet sein, daß die für $p^{2} \rightarrow 0$ unendlich werdenden Teile von $H$, angewandt auf $V$, für $p^{2} \rightarrow 0$ nicht divergieren, während der Rest $R$ mit $p^{2} \rightarrow 0$ selbst verschwinden muß. $V$ muß also im wesentlichen die gleichen Eigenschaften haben wie $\tau(0)$ in $\mathrm{Ab}$ schn. 2 b). Dann trägt nur der Anteil $V$ zu den Kräften langer Reichweite bei. Man erkennt also, daß die Kräfte langer Reichweite im wesentlichen nur durch die ,Lichtquanten“, d. h. durch die Lösungen von (47) übertragen werden, mit dem einzigen Unterschied, daß hier $p^{2} \neq 0$, und zwar positiv, d. h. raumartig und sehr klein $\left(p^{2} \ll x^{2}\right)$ ist.

Für die Darstellung des $V_{\mu f \alpha \varrho}$ ist es zweckmäßig, vier zueinander orthogonale Einheitsvektoren $A_{\varrho}$ einzuführen, von denen der erste parallel zu $p_{\nu}$ ist. Es soll also

$$
A_{\nu}{ }^{1}=p_{\nu} / \sqrt{p^{2}}
$$

gelten. Dann hat der antimetrische Pseudotensor

$$
\gamma_{\varrho} \gamma_{5} \gamma_{\sigma}+\gamma_{\sigma} \gamma_{\varrho} \gamma_{5}
$$

in dem durch die $A_{\varrho}$ aufgespannten Koordinatensystem die Form

$$
T \varrho \sigma=A_{\lambda}^{\varrho} A_{\nu}^{\sigma}\left(\gamma_{\lambda} \gamma_{5} \gamma_{\nu}+\gamma_{\nu} \gamma_{\lambda} \gamma_{5}\right) ;\left(T \varrho \sigma=-T^{\sigma \varrho}\right) \text {. }
$$

Untersucht man nun die Eigenfunktionen $\tau(0)$ für die Bose-Teilchen und verallgemeinert (62) für beliebige Richtungen von $p_{\nu}$, so findet man, da $V_{\mu \beta \alpha \varrho}$ nur Ausdrücke der Form $T_{\mu \lambda}^{\varrho \sigma}$ enthalten kann, da nur diese Ausdrücke die Bedingung (61) befriedigen. In der Tat sind die Eigenfunktionen $\tau(0)$ in $2 \mathrm{~b}$ ) bis auf einen konstanten Faktor mit $T^{1 \sigma}$ identisch, wenn man zum limes $p^{2} \rightarrow 0$ übergeht. Dabei gibt es im limes $p^{2} \rightarrow 0$ nur zwei solche Eigenfunktionen (und nicht, wie gewöhnlich, drei, nämlich $\sigma=2,3,4$ ), weil es zu einem Nullvektor nur zwei orthogonale Vektoren gibt. Man erkennt hier, daß die $A_{\lambda}^{\sigma}$ den Vektorpotentialen der Lichtquanten entsprechen.

Nun kann man das inhomogene Glied in Gl. (92) in folgender Weise umschreiben:

$$
\delta_{\mu \beta} \delta_{\varrho \alpha}=\frac{1}{4} \sum_{N}\left(\gamma_{N}\right)_{\mu \varrho}\left(\gamma_{N}\right)_{\alpha \beta},
$$

wobei $\gamma_{N}$ der Reihe nach alle 16 Elemente der DiracAlgebra bedeuten soll und $\gamma_{N}^{+}=\gamma_{N}$ angenommen wird. Der tensorielle Teil dieser Summe läßt sich nach (96) offenbar auch in der Form

$$
\frac{1}{4} \sum_{\text {tensor. Teil }}\left(\gamma_{N}\right)_{\mu \varrho}\left(\gamma_{N}\right)_{\alpha \beta}=-\frac{1}{16} \sum_{\varepsilon<\eta} T_{\mu \varrho}^{\varepsilon \eta} T_{\alpha \beta}^{\varepsilon \eta}
$$

darstellen. Man erkennt daraus, daß $V$ selbst die Form $V_{\mu \beta \alpha \varrho}=a_{1} \sum_{\eta} T_{\mu \varrho}^{1 \eta} T_{\alpha \beta}^{1 \eta}+\underset{k<l}{a_{2}} \sum_{\mu \varrho} T_{\mu \beta}^{k l} T_{a \beta}^{k l},(k, l \neq 1)$

haben muß, wobei die Zahlenfaktoren $a_{1}, a_{2}$ noch aus (92) zu bestimmen sind. In (92) braucht dabei nur das für $p^{2} \rightarrow 0$ größte Glied in $H$, nämlich

$$
H_{\mu \beta \alpha \varrho}=+2 \pi^{2} \varkappa^{2} \frac{(p \gamma)_{\mu \beta}(p \gamma)_{\alpha \varrho}}{p^{2}}+\ldots,
$$

berücksichtigt zu werden. Durch Einsetzen in (92) folgt

$$
a_{1,2}=-\frac{1}{16\left(1 \mp \frac{(\kappa l)^{2}}{32 \pi^{2}}\right)} .
$$

Damit ist $V$ und bis auf Glieder kleinerer Ordnung auch $Q$ festgelegt. Durch Einsetzen in (93) erhält man

$$
\begin{aligned}
& K_{\mu \varrho / \nu \sigma}=-\frac{i \pi^{2} \varkappa^{4}}{p^{2}} T_{\mu \varrho}^{\varepsilon \tau} \cdot T_{\nu \sigma}^{\eta \lambda} C_{\varepsilon \tau \eta \lambda} \cdot \delta(p+q), \text { wobei } \\
& C_{1 \tau 1 \lambda} \frac{1}{a_{1}^{2}}=T_{\alpha \beta}^{1 \tau} T_{\gamma \delta}^{1 \lambda}\left[\frac{1}{2} \gamma_{\beta \gamma}^{\varrho} \gamma_{\delta \alpha}^{\varrho}\right. \\
& \left.-\frac{(\gamma p)_{\beta \gamma}(\gamma p)_{\delta \alpha}}{p^{2}}+2 \delta_{\alpha \beta} \delta_{\gamma \delta}\right]=16 \delta_{\tau \lambda}-16 \delta_{1 \tau} \delta_{1 \lambda},
\end{aligned}
$$

$\frac{1}{a_{2}{ }^{2}} C_{k l m n}=-16 \delta_{k n} \delta_{l m}+16 \delta_{k m} \delta_{l n} ; C_{1 \tau k l}=C_{k l 1 \lambda}=0$.

Die Glieder mit $\tau=1, \lambda=1$ geben zu (102) keinen Beitrag.

Daher bleibt schließlich

$$
\begin{aligned}
K_{\mu \varrho \mid \nu \sigma}(p, q)= & -\frac{16 i \pi^{2} \varkappa^{4}}{p^{2}} \delta(p+q) \\
& \cdot\left[a_{1}{ }^{2} T_{\mu \sigma}^{1 \tau} T_{\nu \varrho}^{1 \tau}-a_{2}{ }^{2} \Sigma T_{\mu \varrho}^{m n} T_{\nu \sigma}^{m n}\right]
\end{aligned}
$$

Die Übergangsamplitude wird, wieder unter der Voraussetzung $O_{\mu \varrho \mid \eta \xi}^{\mathrm{s}}=\delta_{\eta \mu} \delta_{\varrho \xi}$, nach Ausschreiben der Spinoren $u_{\mu \nu}(p, q \mid)$ unter Ausnützung der Relation $\sum A_{\tau}{ }^{\tau} A_{\nu^{\prime}}{ }^{\tau}=\delta_{\nu \nu^{\prime}}$ : 


$$
\begin{aligned}
A= & \frac{16 i \pi^{2}(\varkappa l)^{4}}{\left(p-p^{\prime}\right)^{2}}\left\{a_{1}^{2}\left[\bar{u}\left(p^{\prime}\right)\left(\left(A^{1} \gamma\right) \gamma_{5} \gamma_{\mu}+\gamma_{\mu}\left(A^{1} \gamma\right) \gamma_{5}\right) u(p)\right]\right. \\
& \left.\cdot\left[\bar{u}\left(q^{\prime}\right)\left(\left(A^{1} \gamma\right) \gamma_{5} \gamma_{\mu}+\gamma_{\mu}\left(A^{1} \gamma\right) \gamma_{5}\right) u(q)\right]-a_{2}{ }^{2} \sum_{m<n}\left[\bar{u}\left(p^{\prime}\right) A_{\varrho}^{m} A_{\sigma}^{n} \gamma_{\sigma \varrho} \gamma_{5} u(p)\right]\left[\bar{u}\left(q^{\prime}\right) A_{\mu}^{m} A_{\nu}^{n} \gamma_{\nu \mu} \gamma_{5} u(q)\right]\right\} \\
& \cdot \delta\left(p-p^{\prime}+q-q^{\prime}\right)-\text { Austauschterm }(p \leftrightarrow q), \quad\left(\gamma_{\varrho \sigma}=\gamma_{\varrho} \gamma_{\sigma}-\gamma_{\sigma} \gamma_{\varrho}\right) .
\end{aligned}
$$

In der Quantenelektrodynamik gehört in der niedrigsten Ordnung zum Graphen $\rangle-\cdots<$ die Übergangsamplitude

$$
\begin{aligned}
A= & \frac{(2 \pi)^{4} i e^{2}}{\left(p-p^{\prime}\right)^{2}}\left[\bar{u}\left(p^{\prime}\right) \gamma_{\mu} u(p)\right]\left[\bar{u}\left(q^{\prime}\right) \gamma_{\mu} u(q)\right] \\
& \cdot \delta\left(p-p^{\prime}+q-q^{\prime}\right) \text { - Austauschterm. }
\end{aligned}
$$

Dabei ist hier, wegen der Benützung der Heavisideschen Einheiten, die Feinstrukturkonstante $\alpha_{\mathrm{F}}=e^{2} / 4 \pi$.

Setzt man die Vorfaktoren in den Ausdrücken der zweiten Zeile von (105) und der rechten Seite von (106) gleich, so ergibt sich

$$
\alpha_{\mathrm{F}}=(x l)^{4} a_{2}^{2} / 4 \pi^{3}=0,068 .
$$

Die Wechselwirkung in unserem Modell unterscheidet sich also in zweierlei Weise von der in der Quantenelektrodynamik. Erstens ist die Feinstrukturkonstante etwa um den Faktor zehn gröBer, zweitens handelt es sich nicht um die übliche vektorielle Wechselwirkung. Man erkennt die Art der Wechselwirkung in (105) am besten, wenn man zum unrelativistischen Grenzfall übergeht, d. h. $p^{2} \ll x^{2}, q^{2} \ll x^{2}$ annimmt und nur die Glieder niedrigster Ordnung in $\mathfrak{p}$ beibehält.

Dann wird

$$
\begin{aligned}
A \approx- & \frac{64 i \pi^{2}(\mathfrak{x} l)^{4}}{\left(\mathfrak{p}-\mathfrak{p}^{\prime}\right)^{2}}\left\{\left(a_{1}{ }^{2}-a_{2}{ }^{2}\right)\right. \\
& \left.\cdot \frac{\left(\sigma^{(1)}, \mathfrak{p}-\mathfrak{p}^{\prime}\right)\left(\sigma^{(2)}, \mathfrak{p}-\mathfrak{p}^{\prime}\right)}{\left(\mathfrak{p}-\mathfrak{p}^{\prime}\right)^{2}}+a_{2}{ }^{2}\left(\sigma^{(1)} \sigma^{(2)}\right)\right\}
\end{aligned}
$$$$
\text { - Austauschterm. }
$$

Es handelt sich also um eine Tensorkraft langer Reichweite. Allerdings kann dieses Resultat noch durch die Näherungsannahme $O_{\mu \varrho \mid \eta \xi}^{\mathrm{s}}=\delta_{\mu \eta} \delta_{\varrho \xi}$ verfälscht sein, die im Rahmen der hier durchgeführten Rechnung nicht konsequent ist. Wenn man nämlich im Operator $L$ über unendilich viele Näherungsschritte summiert, wie es hier geschehen ist, müßte man das gleiche auch bei der Berechnung von $O^{\mathrm{s}}$ tun. An dieser Stelle stößt man auf das Problem der Eichinvarianz, das hier noch nicht angegriffen werden soll und das vielleicht auch erst in einer Theorie behandelt werden kann, die auch den Isotopenspin enthält. Hier sollte nur gezeigt werden, daß die Kräfte langer Reichweite tatsächlich durch das Bosefeld übertragen werden, dessen Teilchen die Lichtquanten sind, und daß sich dabei eine Feinstrukturkonstante $\ll 1$ ergibt.

\section{d) Bose-Teilchen nichtverschwindender Ruhmasse}

Um die Frage der Existenz von Bose-Teilchen nichtverschwindender Ruhmasse im Rahmen des vorliegenden Modells zu untersuchen, sollte man eigentlich mindestens zur Näherung $N=4$ übergehen, da in der Näherung $N=\mathbf{2}$ auch für kleine Energiewerte $\left(J_{\lambda}{ }^{2}<4 \varkappa^{2}\right)$ das Kontinuum der Streuzustände zu erwarten ist, die als Streuung eines Spinorteilchens der Ruhmasse Null an einem anderen derartigen Teilchen interpretiert werden können. Denn erst von $N=3$ ab erhält das einzelne Spinorteilchen seine Ruhmasse.

Trotzdem kann man auch für $N=2$ schon einen ersten Näherungswert wenigstens für einige Eigenwerte des Bosonenspektrums erhalten. Denn die gebundenen Zustände sind von den Streuzuständen leicht dadurch zu unterscheiden, daß die Eigenfunktionen für $z^{2} \rightarrow \infty$ verschwinden, und indirekt ist die Ruhmasse $\varkappa$ der leichtesten Spinorteilchen in der Integralgleichung für $N=2$ durch die $S_{\mathrm{F}}$-Funktion enthalten. Wir wollen also die Massen der Bose-Teilchen doch in der Näherung $N=2$ aus dem $\tau$-Gleichungssystem zu berechnen suchen in der Hoffnung, daß die so gewonnenen Lösungen in der für die Berechnung zu komplizierten Näherung $N=4$ ungefähr erhalten bleiben.

Zur Bestimmung der Eigenwerte $J^{2} \neq 0$ in der Näherung $N=2$ hat man wieder von (47) auszugehen, d. h. von

$$
\begin{aligned}
\tau(z)= & -i l^{2} \int_{\mathrm{F}} \mathrm{d}^{4} p \cdot \frac{\left(-J_{\mu}+p_{\mu}\right)}{\left(-J_{\lambda}+p_{\lambda}\right)^{2}} \gamma_{\mu} \\
& \cdot[-\operatorname{Spur} \tau(0)+\tau(0)] \frac{S(-p)}{2(2 \pi)^{4}} e^{i p_{\sigma^{z} \sigma}} .
\end{aligned}
$$

Das $\mathrm{F}$ am Integralzeichen soll andeuten, daß in der $p_{0}$-Ebene nach der Feynmanschen Vorschrift integriert wird. In dieser Vorschrift ist für $J^{2} \neq 0 \mathrm{im}$ plizite enthalten, daß $\tau(z)$ für $z^{2} \rightarrow \infty$ verschwinden soll. Offenbar ist eine solche Voraussetzung für 
das Zustandekommen eines diskreten Spektrums notwendig.

Aus (109) erhält man für $z=0$ ein System von 16 linearen und homogenen Gleichungen für die $\tau_{\mu \nu}(0)$, woraus man durch Nullsetzen der Determinante die Eigenwerte $J^{2} \neq 0$ findet. Die im Abschnitt 2 b) für den Fall $J^{2}=0$ besprochenen Divergenzen treten hier nicht auf, so daß die Rechnung ohne jeden Umweg ausgeführt werden kann.

Für $S(p) / 2$ gilt die Beziehung

$$
\frac{1}{(2 \pi)^{4}} S(p) / 2=\gamma_{\nu} p_{\nu} F(p)-G(p)
$$

mit

$F(p)=\frac{\chi^{4}}{(2 \pi)^{4} p^{4}\left(p^{2}+\varkappa^{2}\right)}, \quad G(p)=\frac{i \varkappa^{3}}{(2 \pi)^{4} p^{2}\left(p^{2}+\chi^{2}\right)}$.

Damit folgt aus (109) mit $z=0$

$$
\begin{gathered}
\tau(0)=i l^{2}\left\{-J_{\mu} \gamma_{\mu}[-\operatorname{Spur} \tau(0)+\tau(0)] \gamma_{\nu} F_{\nu}(0\}\right. \\
\quad+\gamma_{\mu}[-\operatorname{Spur} \tau(0)+\tau(0)] \gamma_{\nu} F_{\mu \nu}(0) \\
-J_{\mu} \gamma_{\mu}[-\operatorname{Spur} \tau(0)+\tau(0)] G_{0}(0) \\
\left.+\gamma_{\mu}[-\operatorname{Spur} \tau(0)+\tau(0)] G_{\mu}(0)\right\}
\end{gathered}
$$

mit den Abkürzungen

$F_{\nu}(0)=(2 \pi)^{-4} \varkappa^{4} \int_{\mathrm{F}} \frac{\mathrm{d}^{4} p \cdot p_{\nu}}{p^{4}\left(p^{2}+\varkappa^{2}\right)\left(-J_{\lambda}+p_{\lambda}\right)^{2}}$,

$F_{\mu \nu}(0)=(2 \pi)^{-4} \varkappa^{4} \int_{\mathrm{F}} \frac{\mathrm{d}^{4} p \cdot p_{\mu} p_{\nu}}{p^{4}\left(p^{2}+\varkappa^{2}\right)\left(-J_{\lambda}+p_{\lambda}\right)^{2}}$,

$G_{0}(0)=i \varkappa^{3}(2 \pi)^{-4} \int_{\mathrm{F}} \frac{\mathrm{d}^{4} p}{p^{2}\left(p^{2}+\varkappa^{2}\right)\left(-J_{\lambda}+p_{\lambda}\right)^{2}}$,

$G_{\mu}(0)=i \varkappa^{3}(2 \pi)^{-4} \int_{\mathrm{F}} \frac{\mathrm{d}^{4} p \cdot p_{\mu}}{p^{2}\left(p^{2}+\varkappa^{2}\right)\left(-J_{\lambda}+p_{\lambda}\right)^{2}}$.

Die Berechnung der Integrale erfolgt nach den schon mehrfach benützten Feynmanschen Methoden. Dabei ist nur zu bedenken, daß hier $\mathrm{d}^{4} p=\mathrm{d} p_{0} \mathrm{~d} p_{1} \mathrm{~d} p_{2} \mathrm{~d} p_{3}$ ist, im Gegensatz zu der Feynmanschen Bezeichnung $\mathrm{d}^{4} p=(2 \pi)^{-2} \mathrm{~d} p_{0} \mathrm{~d} p_{1} \mathrm{~d} p_{2} \mathrm{~d} p_{3} ; \quad$ und $p^{2}=\overrightarrow{p^{2}}-p_{0}{ }^{2}$ statt $p^{2}=p_{0}^{2}-\overrightarrow{p^{2}}$ wie bei Feynman.

Ferner werden, um kompliziertere Integrale auf einfachere zu reduzieren, die von Feynman benutzte Formel

$$
1 / a b=\int_{0}^{1} \mathrm{~d} z[a z+b(1-z)]^{-2}
$$

und ähnliche, aus ihr durch Differentiationen nach $a$ und $b$ gewonnene Beziehungen verwendet.

Auf diese Weise erhält man die Beziehungen

$G_{0 ; \mu}(0)=\frac{-\varkappa}{16 \pi^{2}} \int_{0}^{1} \mathrm{~d} z \int_{0}^{1} \mathrm{~d} u \frac{\left(1 ; J_{\mu} u\right)}{\alpha u+z}$,

$F_{\nu}(0)=\frac{i J_{\nu}}{16 \pi^{2}} \int_{0}^{1} \mathrm{~d} z(1-z) \int_{0}^{1} \frac{u \mathrm{~d} u}{(\alpha u+z)^{2}}$,

$$
\begin{aligned}
F_{\mu \nu}(0)= & \frac{i}{16 \pi^{2}} \int_{0}^{1} \mathrm{~d} z(1-z) \int_{0}^{1} \frac{\mathrm{d} u}{(\alpha u+z)^{2}} \\
& \cdot\left[\frac{x^{2}}{2} \delta_{\mu \nu}(1-u)(\alpha u+z)+u^{2} J_{\mu} J_{\nu}\right]
\end{aligned}
$$

mit

$$
\alpha=J^{2} / \varkappa^{2} .
$$

Durch Weiterführung der Rechnung erhält man

$$
\begin{aligned}
i l^{2} G_{0}(0) & =C_{0}, \\
i l^{2} G_{\mu}(0) & =-J_{\mu} C_{1}, \\
i l^{2} F_{\nu}(0) & =-J_{\nu} C_{2}, \\
i l^{2} F_{\mu \nu}(0) & =\delta_{\mu \nu} C_{3}+J_{\mu} J_{\nu} C_{4},
\end{aligned}
$$

mit den Abkürzungen

$$
\begin{aligned}
C_{0}= & \frac{-i \varkappa l^{2}}{16 \pi^{2} \alpha}\left[\alpha \lg \left|\frac{1+\alpha}{\alpha}\right|+\lg |1+\alpha|\right], \\
C_{1}= & \frac{i \varkappa l^{2}}{32 \pi^{2} \alpha^{2}}\left[\alpha^{2} \lg \left|\frac{1+\alpha}{\alpha}\right|-\lg |1+\alpha|+\alpha\right], \\
C_{2}= & \frac{l^{2}}{32 \pi^{2}}\left[\frac{1-\alpha^{2}}{\alpha^{2}} \lg |1+\alpha|+\lg \alpha+\frac{1}{\alpha}\right] \\
C_{3}= & \frac{-\varkappa^{2} l^{2}}{192 \pi^{2} \alpha^{2}} \\
& \cdot\left[(1+\alpha)^{3} \lg \left|\frac{1+\alpha}{\alpha}\right|+(1+3 \alpha) \lg |\alpha|-\alpha(1+\alpha)\right], \\
C_{4}= & \frac{-l^{2}}{48 \pi^{2} \alpha^{3}}\left[\alpha(1+\alpha)-\lg |1+\alpha|-\alpha^{3} \lg \left|\frac{1+\alpha}{\alpha}\right|\right] .
\end{aligned}
$$

Für (109) findet man jetzt

$$
\begin{aligned}
\tau(0)= & -\operatorname{Spur} \tau(0)\left[D J^{2}+4 C+B J_{\mu} \gamma_{\mu}\right] \\
& +D J_{\mu} J_{\nu} \gamma_{\mu} \tau(0) \gamma_{\nu}+C \gamma_{\mu} \tau(0) \gamma_{\mu}+B J_{\mu} \gamma_{\mu} \tau(0),
\end{aligned}
$$

wobei

$$
-B=C_{0}+C_{1}, C=C_{3}, D=C_{2}+C_{4} .
$$

Um die Determinante des Systems bequem hinschreiben zu können, scheint es zweckmäßig zu sein, zu anderen Veränderlichen

$$
T_{\varrho}(0)=O_{\varrho} \tau(0)
$$

überzugehen, wo die Operatoren $O(\varrho=1,2,3, \ldots, 16)$ der Reihe nach Multiplikation mit 1, $\gamma_{1}, \gamma_{2}, \gamma_{3}, \gamma_{4}, \gamma_{1} \gamma_{2}$, $\gamma_{1} \gamma_{3}, \gamma_{1} \gamma_{4}, \gamma_{2} \gamma_{3}, \gamma_{2} \gamma_{4}, \gamma_{3} \gamma_{4}, \gamma_{1} \gamma_{2} \gamma_{3}, \gamma_{1} \gamma_{2} \gamma_{4}, \gamma_{1} \gamma_{3} \gamma_{4}, \gamma_{2} \gamma_{3} \gamma_{4}$, $\gamma_{5}$ mit darauf folgender Spurenbildung bedeuten. Es ist leicht einzusehen, daß umgekehrt die $\tau_{\mu \nu}(0)$ durch die $T_{\varrho}(0)$ eindeutig bestimmt sind. Aus (130) folgt nun, indem beide Seiten mit $O_{\varrho}$ multipliziert werden,

$$
\begin{aligned}
T_{\varrho}(0)= & -T_{1}(0) O_{\varrho}\left[D J^{2}+4 C+B J_{\mu} \gamma_{\mu}\right] \\
& +D O_{\varrho}\left[J_{\mu} J_{\nu} \gamma_{\mu} \tau(0) \gamma_{\nu}\right] \\
& +C O_{\varrho}\left[\gamma_{\mu} \tau(0) \gamma_{\mu}\right]+B O_{\varrho}\left[J_{\mu} \gamma_{\mu} \tau(0)\right] .
\end{aligned}
$$

Von jetzt ab wird $J_{1}=J_{2}=J_{3}=0$ angenommen, was keine Einschränkung der Allgemeinheit bedeutet, aber die Rechnung vereinfacht. Nach Durchführung dieser Transformation findet man, daß jetzt das System (133) 
in acht Einzelsysteme mit je zwei Unbekannten $T_{\varrho}(0)$ zerfällt, welche wie folgt lauten, wenn die $T_{\varrho}(0)$ einfach mit $T_{\varrho}$ bezeichnet werden:

$$
\begin{aligned}
& T_{1}\left[-3 D J^{2}-12 C-1\right]+T_{5} B J_{4}=0, \\
& T_{1}\left(-3 B J_{4}\right)+T_{5}\left[D J^{2}-2 C-1\right]=0 ; \\
& T_{4}\left[-D J^{2}-2 C-1\right]+T_{11} B J_{4}=0, \\
& T_{4} \cdot B J_{4}+T_{11}\left[-D J^{2}-1\right]=0 ; \\
& T_{2}\left[-D J^{2}-2 C-1\right]+T_{8} B J_{4}=0, \\
& T_{2} B J_{4}+T_{8}\left[-D J^{2}-1\right]=0 ; \\
& T_{7}\left[D J^{2}-1\right]+T_{14} B J_{4}=0, \\
& T_{7} B J_{4}+T_{14}\left[D J^{2}+2 C-1\right]=0 ; \\
& T_{3}\left[-D J^{2}-2 C-1\right]+T_{10} B J_{4}=0, \\
& T_{3} B J_{4}+T_{10}\left[-D J^{2}-1\right]=0 ; \\
& T_{9}\left[D J^{2}-1\right]+T_{15} B J_{4}=0, \\
& T_{9} B J_{4}+T_{15}\left[D J^{2}+2 C-1\right]=0 ; \\
& T_{6}\left[D J^{2}-1\right]+T_{13} B J_{4}=0, \\
& T_{6} B J_{4}+T_{13}\left[D J^{2}+2 C-1\right]=0 ; \\
& T_{12}\left[-D J^{2}+2 C-1\right]+T_{16} B J_{4}=0, \\
& T_{12} B J_{4}+T_{16}\left[-D J^{2}-4 C-1\right]=0 .
\end{aligned}
$$

Die Determinanten dieser acht Systeme hängen nur von der Invarianten $J^{2}$ ab und werden im folgenden mit $D_{1}(\alpha), D_{2}(\alpha), \ldots, D_{8}(\alpha)\left(\alpha=J^{2} / \varkappa^{2}\right)$ bezeichnet. Man sieht sofort, daß

$D_{2}(\alpha)=D_{3}(\alpha)=D_{5}(\alpha)$ und $D_{4}(\alpha)=D_{6}(\alpha)=D_{7}(\alpha)$.

Es bleiben also nur vier verschiedene Determinanten übrig, für die sich die Ausdrücke

$D_{1}(\alpha)=-\left(D J^{2}-2 C-1\right)\left(3 D J^{2}+12 C+1\right)+3 B^{2} J^{2}$,

$D_{2}(\alpha)=\left(D J^{2}+1\right)\left(D J^{2}+2 C+1\right)-B^{2} J^{2}$,

$D_{4}(\alpha)=\left(D J^{2}-1\right)\left(D J^{2}+2 C-1\right)-B^{2} J^{2}$,

$D_{8}(\alpha)=$$$
\left(D J^{2}+4 C+1\right)\left(D J^{2}-2 C+1\right)-B^{2} J^{2}
$$

ergeben.

Zur Bestimmung der Eigenwerte $J^{2} \neq 0$ erhält man also die vier Gleichungen

$$
\begin{aligned}
& D_{1}(\alpha)=0, \\
& D_{2}(\alpha)=0, \\
& D_{4}(\alpha)=0, \\
& D_{8}(\alpha)=0 .
\end{aligned}
$$

Man wird wegen (134) erwarten, daß die zu (139) u. (142) gehörigen Teilchen den Spin 0 besitzen, während die Lösungen von (140) u. (141) zum Spin 1 gehören. Man kann dies auch durch Ausrechnen der Drehimpulse verifizieren.

Die Auflösung der Gln. (139) bis (142) wird wohl nur auf graphischem oder numerischem Wege gelingen. Um die Behandlung zu erleichtern, wird

$B^{2} J^{2}=\frac{-\lambda^{4}}{\left(32 \pi^{2}\right)^{2}} \beta, \quad C=\frac{-\lambda^{2}}{192 \pi^{2}} \gamma, \quad D J^{2}=\frac{-\lambda^{2}}{96 \pi^{2}} \delta$

gesetzt, wo

$$
\lambda=\varkappa l=7,426 .
$$

Für $\beta, \gamma, \delta$ findet man dann nach (126) bis (129) und (131)

$\beta=\frac{\left[\alpha+\alpha^{2} \lg \alpha-(1+\alpha)^{2} \lg |1+\alpha|\right]^{2}}{\alpha^{3}}$,

$\gamma=\frac{(1+\alpha)^{3} \lg |1+\alpha|}{\alpha^{2}}-(\alpha+3) \lg |\alpha|-1-\frac{1}{\alpha}$,

$\delta=\alpha \lg \left|1+\frac{1}{\alpha}\right|-1+\frac{2}{\alpha}-\frac{(2+3 \alpha)}{\alpha^{2}} \lg |1+\alpha|$,

und die Gln. (139) bis (142) lauten jetzt

$$
\begin{aligned}
& -D_{1}(\alpha)=-[0,175(\delta+2 \gamma)-1][0,058(\gamma-\delta)-1] \\
& +0,0918 \beta=0 \text {, } \\
& D_{2}(\alpha)=[1-0,0583 \delta][1-0,0583(\gamma+\delta)] \\
& +0,0306 \beta=0 \\
& -D_{4}(\alpha)=[0,058 \delta+1][1+0,058(\gamma+\delta)] \\
& +0,03 \beta=0 \text {, } \\
& D_{8}(\alpha)=-[1+0,0583(\gamma-\delta)][0,0583(\delta+2 \gamma)-1] \\
& +0,0306 \beta=0 \text {. }
\end{aligned}
$$

(148) hat die zwei Lösungen

$$
\alpha_{1}(1)=-0,0165, \alpha_{1}{ }^{(2)}=-0,20 .
$$

(149) hat nur die Lösurng

$$
\alpha_{2}=-0,002 \text {, }
$$

während (150) keine besitzt. Endlich hat (151) die Nullstelle

$$
\alpha_{8}=-0,055 \text {. }
$$

Wegen $\alpha=J^{2} / \varkappa^{2}=-m^{2} / \varkappa^{2}$ ergeben sich für die zugehörigen Ruhmassen der Bosonen, welche bzw. mit $m_{1}{ }^{(1)}, m_{1}{ }^{(2)}, m_{2}, m_{8}$ bezeichnet werden, die Werte $m_{1}{ }^{(1)}=0,95 / l, m_{1}{ }^{(2)}=3,32 / l, m_{2}=0,33 / l, m_{8}=1,74 / l$.

Die angegebenen Werte sind nur auf einige Prozente genau; auf größere Genauigkeit ist kein Wert gelegt worden.

Vergleicht man diese Werte mit der Masse $\varkappa=7,4 / l$ des Spinorteilchens, so erkennt man, daß die Massen der leichtesten Bosonen um einen Faktor der Größenordnung zehn kleiner sind, als die des Spinorteilchens, das mit dem Nukleon verglichen werden muß. In dieser Hinsicht zeigt das Eigenwertspektrum also eine bemerkenswerte Ähnlichkeit mit dem der wirklichen Elementarteilchen. Das Teilchen mit der Masse $m_{2}$ besitzt den Spin 1: die übrigen den Spin 0 .

\section{e) Die Integralgleichungen für die Funktion $S_{\mathrm{F}}$}

Die Integralgleichungen, die in den vorhergehenden Abschnitten zur Berechnung von $\tau$-Funktionen benützt worden sind, müssen auch für die $S_{\mathrm{F}^{-}}$ 
Funktion selbst gelten, da ja $1 / 2 S_{F}$ nach der Definition identisch ist mit der $\tau$-Funktion von zwei Variabeln, die zum Übergang vom Vakuum zum Vakuum gehört. Die Integralgleichungen für $S_{\mathrm{F}}$ sind aber im Gegensatz zu den bisherigen nichtlinear, da $S_{\mathrm{F}}$ auch in dem durch das Graphenschema dargestellten Kern $K$ vorkommt. Wenn man $S_{F}$, wie dies bisher geschehen ist, nach Gl. (48) durch den Beitrag des tiefsten Eigenzustandes approximiert, so können die Integralgleichungen, die sich z. B. in der Näherung $N=4$ ergeben, nur näherungsweise erfüllt sein. Wir wollen nun speziell die Näherung $N=4$ zum Ausgangspunkt für eine wenigstens qualitative Nachprüfung der Annahmen über die $S_{\mathrm{F}}$-Funktion machen.

Der allgemeine Typus der Integralgleichung für $S_{\mathrm{F}}$ in der Näherung $N=4$ kann etwa durch

$$
\gamma_{\mu} \frac{\partial S_{\mathrm{F}}}{\partial z_{\mu}}=G_{\mathrm{F}} \cdot S_{\mathrm{F}}{ }^{3}
$$

angedeutet werden. Die Ähnlichkeit mit der Ausgangswellengleichung (1) macht es wahrscheinlich, daß sich aus dieser Gleichung in der Nähe des Lichtkegels ein oszillierendes Verhalten für $S_{\mathbf{F}}$ ergibt, wie es in 1. c. II für Gl. (1) nachgewiesen wurde. In höheren Näherungen sollte das Oszillationsgebiet immer näher an den Lichtkegel heranrücken. Daraus geht aber bereits hervor, daß die Näherungslösung [Gl. (48) oder 1. c. II, Gln. (31) und (33)], die bisher für $S_{\mathrm{F}}$ benützt worden ist, in unmittelbarer Nähe des Lichtkegels die Integralgleichung nicht erfüllen kann; denn in dieser Näherungslösung ist durch die Annahme $S(0)=0$ bereits über die Oszillationen gemittelt. Es muß also als eine ausreichende Übereinstimmung angesehen werden, wenn die Lösung der Integralgleichung in einigem Abstand vom Lichtkegel den bisherigen Ansätzen [Gl. (48)] nahekommt und in der Nähe des Lichtkegels Oszillationen zeigt, die zu einem Integral über die $S_{\mathrm{F}}$-Funktion nur wenig beitragen. Sehr viel besser kann aber der entscheidende Punkt der Theorie, nämlich der Ersatz der $\delta$-Funktionen auf dem Lichtkegel durch die unendlichen Oszillationen und damit ihr Weglassen, an dem Verhalten der $S_{\mathrm{F}}$-Funktion für große raumartige Abstände kontrolliert werden. Der schon in der Einleitung ausführlich besprochene langsame Abfall der $S_{\mathrm{F}^{-}}$bzw. $S_{1}$-Funktion bei großen raumartigen Abständen ist ja wegen der Beziehung (6) eine unmittelbare Folge des Weglassens der $\delta$ Funktionen. Es kann also nachgeprüft werden, ob dieser langsame Abfall mit den nichtlinearen Integralgleichungen verträglich ist. In der Näherungslösung (48) ist der Abfall durch

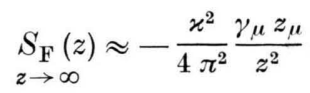

gegeben. In der exakten $S_{\mathrm{F}}$-Funktion muß die $\mathrm{Ab}$ hängigkeit von $z_{\mu}$ die gleiche sein, jedoch wird an die Stelle von $\varkappa^{2}=(7,4 / l)^{2}$ ein gewogener Mittelwert über die Massen verschiedener Eigenzustände treten, von denen $\varkappa$ der tiefste ist. Es muß also jetzt die Frage untersucht werden, ob die nichtlinearen Integralgleichungen für große Werte von $z$ durch

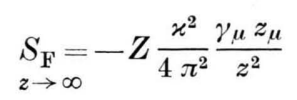

befriedigt werden können, wobei $Z$ ein zu bestimmender Zahlfaktor ist.

Die möglichen Integralgleichungen sind wie in la) und 1 b) durch die Wahl der G-Linien festgelegt und zerfallen, wie die nähere Untersuchung zeigt, in der Näherung $N=4$ in zwei Gruppen, die schematisch durch die beiden Bilder Abb. 18 a und $b$ beschrieben werden können.

Die Integralgleichungen der ersten Gruppe sind nicht besonders interessant, da in ihnen die Wechselwirkung nur indirekt eine Rolle spielt. Sie sind,

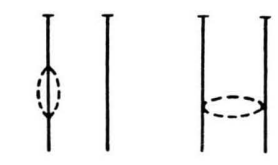

Abb. 18a. Abb. 18b. wenn man sie für die Funktionen $S_{\mathrm{F}}(p)$ aufgeschrieben denkt, durch die Näherung (48) in der unmittelbaren Umgebung von $p_{\mu}{ }^{2}=-\varkappa^{2}$ exakt erfüllt, wenn $\varkappa$ der durch die linke Hälfte des Graphen berechnete Eigenwert $\varkappa=7,4 / l$ ist, in anderen Gebieten des $p$-Raumes sind sie entsprechend schlecht erfüllt; insbesondere lassen diese Integralgleichungen sich kaum für die Kontrolle des asymptotischen Verhaltens (158) verwenden.

Die andere Integralgleichung, die zu Abb. 18b gehört, enthält die Wechselwirkung ausdrücklich; wir schreiben sie nicht als Integralgleichung, sondern in differentieller Form im Anschluß an 1. c. II, G. (62):

$$
\begin{aligned}
& -\frac{1}{2} \gamma_{\mu} \frac{\partial S_{\mathrm{F}}(-z)}{\partial z_{\mu}}=-l^{2} \sigma(z) \\
\frac{\partial \sigma(z)}{\partial z_{\mu}} \gamma_{\mu}= & \frac{l^{2}}{8}\left[S_{\mathrm{F}}(-z) \operatorname{Spur}\left(S_{\mathrm{F}}(z) S_{\mathrm{F}}(-z)\right)\right. \\
& \left.-S_{\mathrm{F}}(-z) S_{\mathrm{F}}(z) S_{\mathrm{F}}(-z)\right] .
\end{aligned}
$$

Durch Einsetzen von (158) erhält man unmittelbar: 


$$
\begin{aligned}
\sigma(z) & =Z \varkappa^{2} / 4 \pi^{2} l^{2} z^{2} \text { und } \\
-\frac{Z \varkappa^{2} \gamma_{\mu} z_{\mu}}{2 \pi^{2} l^{2} z^{4}} & =-\frac{3}{8} \frac{l^{2} \varkappa^{6} Z^{3}}{\left(4 \pi^{2}\right)^{3}} \frac{\gamma_{\mu} z_{\mu}}{z^{4}}, \text { d. h. } \\
(l \varkappa / 4 \pi)^{4} \cdot Z^{2} & =1 / 3 \text { und aus }(32) \\
Z^{2} & =8,35 / 3 ; Z=1,67 .
\end{aligned}
$$

Man erkennt also, daß die Differentialgln. (159) und (160) durch das asymptotische Verhalten (158) tatsächlich befriedigt werden. Der Zahlwert von $Z$ deutet an, daß der gewogene Mittelwert über die Quadrate aller Massen von Fermionen etwa um den Faktor 1,67 höher liegt als das Quadrat der niedrigsten Masse, was durchaus plausibel ist. Damit ergibt sich indirekt eine wenn auch nur qualitative Bestätigung der Lage des tiefsten Eigenwerts.

Der letzte Abschnitt 2 e) zeigt durch das Studium der die Funktion $S_{F}$ bestimmenden Integralgleichungen, daß die Theorie die innere Konsistenz zu besitzen scheint, die für die Konvergenz des ganzen Verfahrens eine notwendige Voraussetzung darstellt. Der Beweis für die Konvergenz und damit für die Existenz von Lösungen des vollständigen Gleichungssystems ist allerdings bisher nicht versucht worden und würde wohl auch mathematische Mittel erfordern, die über das Bisherige weit hinausgehen. Andererseits zeigen die vorliegenden Rechnungen, daß die zugrunde gelegte Theorie im Falle der Konvergenz die in den üblicher Theorien auftretenden Schwierigkeiten vermeidet. Es gibt hier keine unendlichen Größen, die erst renormiert werden müßten, die $S$-Matrix ist sicher unitär und die Eigenzustände besitzen positive Massen und Energien. Auch hinsichtlich der Kausalitätsforderungen der speziellen Relativitätstheorie sind keine Schwierigkeiten zu erkennen; von dem nichtlokalen Verhalten, das in den üb- lichen Theorien aus dem Verschwinden der Unendlichkeiten auf dem Lichtkegel folgt, scheinen hier nur die Kräfte langer Reichweite übriggeblieben zu sein, die als genaues modellmäßiges Abbild der elektromagnetischen Kräfte keine physikalischen Schwierigkeiten bereiten sollten. Allerdings wäre zu einem vollen Verständnis dieser Kräfte noch zu untersuchen, wie es in der Modelltheorie mit der Eichinvarianz und der Erhaltung der Ladung steht. Eine Beantwortung dieser Fragen steht noch aus.

Das Eigenwertspektrum der Gl. (1) unterscheidet sich zwar von dem Massenspektrum der wirklichen Elementarteilchen, ist ihm aber doch in den qualitativen Zügen überraschend ähnlich. Das Fermion mit der Masse $\varkappa=7,4 / l$ muß mit dem Nukleon verglichen werden. Die im Abschnitt 2d) berechneten Bosonen entsprechen verschiedenen Mesonenarten. Der erste Eigenwert der Bosonen ist auch hier um einen Faktor der Größenordnung zehn kleiner als die Masse der Fermions und kann daher mit dem $\pi$-Meson verglichen werden. Die höheren Eigenwerte entsprechen in ihrer Lage etwa den $\tau$ - oder allgemein den K-Mesonen. $\mathrm{Zu}$ Elektron und Neutrino und dem Isotopenspin gibt es in dem Modell kein Analogon. Jedoch treten eben jene Kräfte langer Reichweite zwischen den Elementarteilchen auf, die den elektromagnetischen aufs engste verwandt sind, und die zugehörigen Bosonen der Kuhmasse Null. Die Feinstrukturkonstante ist in der berechneten Näherung etwa 1/15. Auf Grund dieser qualitativen Ähnlichkeit des Eigenwertspektrums der Modelltheorie mit dem wirkliches: Massenspektrum dürfte es sich lohnen, den in der Modelltheorie ausgedrückten mathematischen Möglıcnkeiten für eine Theorie der Elementarteilch $\$ 1$ noch weiter nachzugehen, was in späteren Untersuchungen erfolgen soll. 